\title{
How spatial heterogeneity affects transient behavior in reaction-diffusion systems for ecological interactions
}

\author{
Xiaoying Wang \\ Department of Mathematics and Statistics, University of Ottawa \\ Ottawa, ON K1N 6N5, Canada \\ Messoud Efendiev \\ Helmholtz Center Munich, Institute of Biomathematics and Biostatistics \\ Ingolstädter, Landstrasse 1, 85764 Neuherberg, Germany \\ Frithjof Lutscher \\ Department of Mathematics and Statistics, and Department of Biology, University of Ottawa \\ Ottawa, ON K1N 6N5, Canada
}

\begin{abstract}
Most studies of ecological interactions study asymptotic behavior, such as steady states and limit cycles. The transient behavior, i.e., qualitative aspects of solutions as and before they approach their asymptotic state, may differ significantly from asymptotic behavior. Understanding transient dynamics is crucial to predicting ecosystem responses to perturbations on short time scales. Several quantities have been proposed to measure transient dynamics in systems of ordinary differential equations. Here, we generalize these measures to reactiondiffusion systems in a rigorous way and prove various relations between the non-spatial and spatial effects, as well as an upper bound for transients. This extension of existing theory is crucial for studying how spatially heterogeneous perturbations and the movement of biological species involved affect transient behaviors. We illustrate several such effects with numerical simulations.
\end{abstract}

Keywords: Reaction-diffusion systems; transient dynamics; spatially heterogeneous perturbations.

Mathematics Subject Classification: 35K57; 35B35.

\section{Introduction}

A fundamental question in conservation management is how stable ecological communities react to the one-time perturbations to which they are subject, either from natural or human disturbances, on different spatial scales. For example, marine systems may experience catastrophic 
events such as hurricanes over relatively large scales, whereas other disturbances such as the passing of a harvesting vessel occur on smaller scales. Similarly, in near-shore systems, a localized weather event or a chemical spill inland may cause a localized perturbation at the mouth of a river, whereas near-shore weather-induced flooding or annual fisheries could affect a greater spatial extent (Bailey and Secor 2016; Carpenter and Brock 2004; Matthews 1986). In all cases, there is a one-time perturbation that affects an otherwise stable community, and the intensity of the perturbation may vary spatially.

One longstanding focus of studies in ecosystem management is the asymptotic behavior of a system: how long will it take for the system return to its original state after a perturbation? One measure for this return time to steady state stems from the resilience of a system (Pimm and Lawton 1977). However, even a highly resilient ecological system may experience long transient behavior before reaching its asymptotic steady state, and this transient behavior may differ significantly from the asymptotic behavior (Neubert and Caswell 1997). Transient dynamics have been studied in many non-spatial settings (Hastings 2004; Hastings et al. 2018; Bailey and Secor 2016; Matthews 1986).

We illustrate some of the possible spatial aspects of transient behavior with a spatially distributed predator-prey system. We denote by $\omega_{1}(x, t)$ the density of the prey species that diffuses, grows logistically and gets consumed by a specialist predator, denoted by $\omega_{2}(x, t)$, where $t$ and $x$ indicate time and one-dimensional space, respectively. Our model system reads (in non-dimensional form)

$$
\begin{aligned}
& \frac{\partial \omega_{1}(x, t)}{\partial t}=r_{1} \omega_{1}\left(1-\omega_{1}\right)-\omega_{1} \omega_{2}+d_{1} \frac{\partial^{2} \omega_{1}}{\partial x^{2}} \\
& \frac{\partial \omega_{2}(x, t)}{\partial t}=\theta \omega_{1} \omega_{2}-\omega_{2}+d_{2} \frac{\partial^{2} \omega_{2}}{\partial x^{2}}
\end{aligned}
$$

where $r_{1}$ is the prey growth rate, $\theta$ is the conversion efficiency, and $d_{i}$ are the respective diffusion coefficients. We consider this system on a bounded one-dimensional domain with no-flux boundary conditions. Then there is a positive coexistence steady state $\left(\omega_{1}^{*}, \omega_{2}^{*}\right)$ when $\theta>1$. This state is spatially constant. It is locally (and even globally) asymptotically stable. We write $\omega_{i}=\omega_{i}^{*}+\tilde{\omega}_{i}$ and linearize (1.1) at the positive steady state to obtain an equation for the small perturbation $\tilde{\omega}_{i}$, as usual. We drop the ${ }^{\sim}$ for notational convenience and obtain the system

$$
\begin{aligned}
& \frac{\partial \omega_{1}(x, t)}{\partial t}=J_{11} \omega_{1}+J_{12} \omega_{2}+d_{1} \frac{\partial^{2} \omega_{1}}{\partial x^{2}} \\
& \frac{\partial \omega_{2}(x, t)}{\partial t}=J_{21} \omega_{1}+J_{22} \omega_{2}+d_{2} \frac{\partial^{2} \omega_{2}}{\partial x^{2}}
\end{aligned}
$$

where $J_{i j}$ correspond to entries of the Jacobian matrix from linearizing the nonspatial model underlying (1.1). In particular, since we model a predator-prey system, we have $J_{12}<0$ and 
$J_{21}>0$. Since the positive steady state is stable, all solutions of (1.2) will eventually decay to zero. Figure 1 shows that this eventual decay can be preceded by an initial increase in one or both state variables, and that the increase (or not) depends on initial conditions. Specifically, we chose two initial conditions, one spatially spread out $\left(\hat{\boldsymbol{\omega}}_{0}^{1}\right)$, the other spatially concentrated $\left(\hat{\boldsymbol{\omega}}_{0}^{2}\right)$, both with the same magnitude (according to the $L_{2}$-norm). Figure 1 (a) shows that with the spread-out initial conditions, the density of the predator increases initially everywhere in the domain before it decays to zero. We say that this perturbation is amplified. In contrast, Figure 1(b) shows that with the spatially concentrated initial conditions, the peak density decreases monotonically in time (attenuates). More interestingly, Figure 1(c) shows that the density of the predator increases at other locations where the initial perturbation is almost zero. The prey densities following both perturbations decrease monotonically to zero at every location and thus the plots are omitted here. Figure 1(d) shows the $L_{2}$ norms of solutions following different perturbations (see definition and illustration in the next section).

The observations in Figure 1 have clear relevance in conservation and management. Perturbations of equal magnitude may affect a system quite differently, depending on how they are spatially distributed. The time scales of amplification and attenuation are clearly relevant in real-life applications, so that these transients cannot be ignored (Hastings 2004; Hastings et al. 2018; Neubert and Caswell 1997). In this work, we study two particular measures of transient behavior: the reactivity and the amplification envelope for systems of reaction-diffusion equations.

The concepts of reactivity and amplification envelope were first introduced and studied in ecological systems by Neubert and Caswell (1997) in the context of ordinary differential equations. The concepts apply only for systems where a local positive steady state exists. One assumes that the state of the system is perturbed away from that steady state and characterizes the return of the system to the steady state. When all perturbations decay initially (attenuate), the system is called non-reactive. When some perturbations grow initially (amplify), the system is called reactive. More formally, reactivity is defined as the maximum relative growth rate of solutions immediately after a single (small) perturbation. We give the explicit formula below. The amplification envelope measures how the maximum relative size of a perturbation evolves in time before it approaches zero. Neubert and Caswell showed that a variety of ecological interactions lead to reactive systems. In particular, small perturbations of existing ecosystems can, in the short-term, produce unexpectedly large deviations from a long-term observed state of the system. Subsequent studies extended these concepts to discrete-time systems (Caswell and Neubert 2005) and related reactivity to necessary conditions for diffusive instability (Neubert et al. 2002).

More recently, the study of transient dynamics has attracted attention of researchers from different aspects. Arnoldi et al. (2016) studied transient behavior induced by continuous-time 
stochastic perturbations. Mari et al. (2017) extended the results in Neubert and Caswell (1997) to a more general definition of reactivity. Generalized reactivity measures the transient growth of a perturbation on specific state variables, different from the definition in Neubert and Caswell (1997), where the perturbation is imposed on all state variables. Vesipa and Ridolfi (2017) examined transients of non-autonomous systems which are subjected to seasonal forcing. More theoretical analysis and detailed study of transient dynamics can also be found in Verdy and Caswell (2008); Wang et al. (2017). From the experimental aspect, transients have been studied numerically for a variety of ecological systems; see Snyder (2010); Stott et al. (2011); Stott (2016); Townley et al. (2007) for examples.

One aspect that has been largely ignored in studies of transients is the impact and importance of spatial location and profile of a perturbation. One of the difficulties with spatially extended systems is that the state space becomes infinite dimensional. We only know of two studies that address spatial aspects of transient dynamics. Anderson et al. (2008) study transient dynamics following spatial perturbations in advective systems such as rivers or streams. The authors take Fourier transforms of the linearized equations, which leads to a finite-dimensional system for each Fourier mode. The authors then study reactivity and amplification envelopes following and extending the finite-dimensional theory from Neubert and Caswell (1997) for each mode. They thereby link the reactivity and the amplification envelope to the spatial wave length of a perturbation. Since they study stream ecosystems, they relate these quantities to the socalled response length that indicates how far downstream a perturbation is felt. A different approach to measure spatial transients was taken by Phillips (2017). The author considers an integrodifference model for population dynamics on a moving habitat. The author discretizes the habitat and applies the corresponding finite-dimensional theory from Caswell and Neubert (2005) to the discretized finite dimensional system. The author explores how different distribution kernels affect transient behavior and shows that transients can differ by an order of magnitude from asymptotic behavior (Phillips 2017).

In this paper, we work with the reaction-diffusion system directly. We extend the previous definitions and results of reactivity and amplification envelope to reaction-diffusion systems, thereby putting the study of spatial effects of these two quantities on a solid theoretical footing. In Section 2.1, we set up the general mathematical framework required for the analysis. We define reactivity and derive an explicit formula to calculate reactivity in Section 2.2. In Section 2.3, we give an upper bound of the amplification envelope. One of the main results is that reactivity is independent of spatial configurations. However, the transient behavior of spatially heterogeneous perturbations shows some systematic patterns. We run numerical simulations to illustrate our theoretical results and further explore the role that spatially heterogeneous distribution has on transients. We end the paper by summarizing conclusions and discussions. 


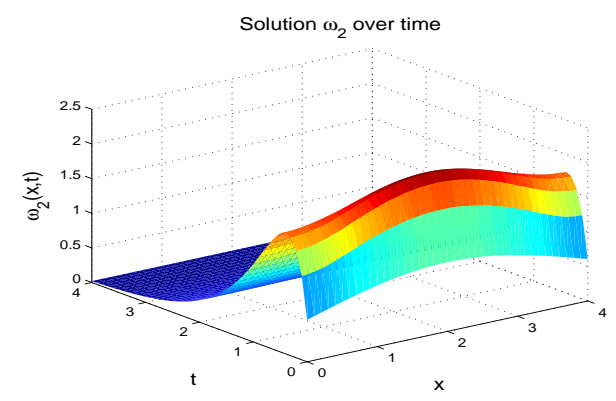

(a) Perturbation to the predator density following $\hat{\boldsymbol{\omega}}_{0}^{1}$

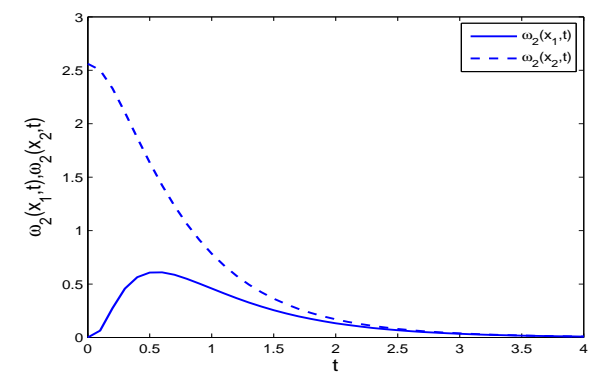

(c) Solution $\omega_{2}(x, t)$ following $\hat{\boldsymbol{\omega}}_{0}^{2}$ at different locations

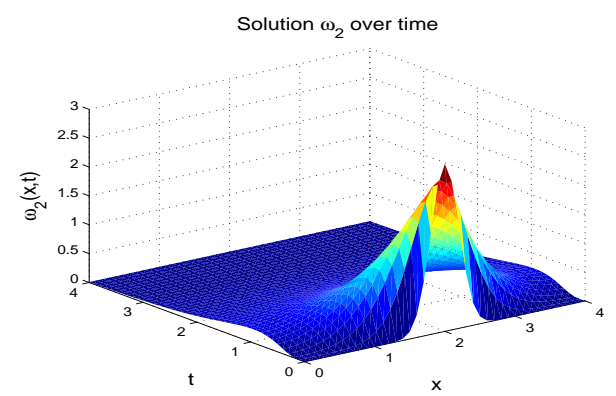

(b) Perturbation to the predator density following $\hat{\boldsymbol{\omega}}_{0}^{2}$

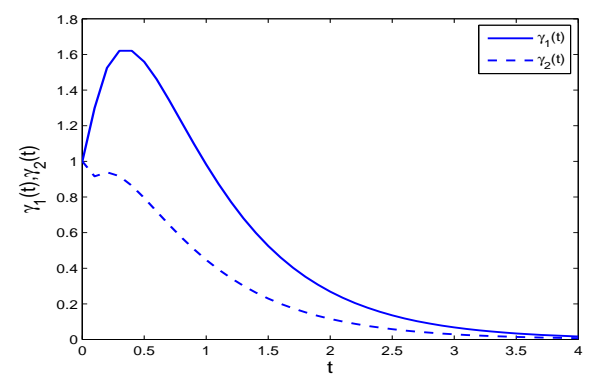

(d) $L_{2}$ norms of solutions following $\hat{\boldsymbol{\omega}}_{0}^{1}, \hat{\boldsymbol{\omega}}_{0}^{2}$

Figure 1: Evolution of perturbations to the spatial predator-prey model (1.2). Figures 1(a) and $1(\mathrm{~b})$ show the perturbations to the predator density as time evolves, following different initial data. Figure 1(c) further shows the perturbation to the predator density as time evolves, at different spatial locations. Figure $1(\mathrm{~d})$ shows the $L_{2}$ norm of solutions following different initial data (for precise definitions of $\gamma_{j}$, see (3.2)). Parameters are: $J_{11}=-5, J_{12}=-0.5, J_{21}=$ $10, J_{22}=0, d_{1}=1, d_{2}=0.5$. We denote different initial data of $(1.2)$ as $\hat{\boldsymbol{\omega}}_{0}^{j}=\left(\omega_{1}^{j}(x, 0), \omega_{2}^{j}(x, 0)\right)=$ $\left(\omega_{10}^{j}, \omega_{20}^{j}\right)$, where $j=1,2, \cdots$. Initial data are $\omega_{10}^{1}=\omega_{20}^{1}=\exp \left[-\left((x-2)^{2}\right) /\left(2\left(2^{2}\right)\right)\right]$ and $\omega_{10}^{2}=$ $\omega_{20}^{2}=2.9026 \exp \left[-\left((x-2)^{2}\right) /\left(2\left(0.2^{2}\right)\right)\right]$. 


\section{Transient Dynamics of Reaction-Diffusion Systems}

We analyze short-term dynamics of spatial systems where solutions tend to a spatially homogeneous steady state asymptotically. Because of the long-term convergence, the linearized system at the steady state provides a good approximation of the original nonlinear system, assuming that perturbations around the steady state are small.

\subsection{General Model Framework}

Based on the above assumption, we first consider a general model, comprised of $n$ populations that interact locally and diffuse randomly within a habitat, as

$$
\begin{aligned}
\frac{\partial \omega_{1}}{\partial t} & =g_{1}\left(\omega_{1}\right)+f_{1}\left(\omega_{1}, \omega_{2}, \cdots, \omega_{n}\right)+d_{1} \Delta \omega_{1} \\
\frac{\partial \omega_{2}}{\partial t} & =g_{2}\left(\omega_{2}\right)+f_{2}\left(\omega_{1}, \omega_{2}, \cdots, \omega_{n}\right)+d_{2} \Delta \omega_{2} \\
& \vdots \\
\frac{\partial \omega_{n}}{\partial t} & =g_{n}\left(\omega_{n}\right)+f_{n}\left(\omega_{1}, \omega_{2}, \cdots, \omega_{n}\right)+d_{n} \Delta \omega_{n} .
\end{aligned}
$$

In the above model, $\omega_{i}(x, t)$ for $1 \leq i \leq n$ represents the density of species $i$ at spatial location $x$ and time $t$. Functions $g_{i}$ and $f_{i}$ represent intraspecific and interspecific interactions, respectively. Parameter $d_{i}$ represents the random diffusion rate of species $i$, and $\Delta$ is the Laplacian operator. We consider a bounded domain $\Omega \subset \mathbb{R}^{N}$ and impose Neumann boundary conditions for $\omega_{i}(x, t)$ such that

$$
\frac{\partial \omega_{i}(x, t)}{\partial \nu}=0, \quad \text { for } \quad x \in \partial \Omega
$$

where $\nu$ is the outward normal vector. Moreover, $\omega_{i}(x, t)$ satisfy continuous initial data $\omega_{i}(x, 0)=$ $\omega_{i 0}(x)$.

We assume that solutions of (2.1) asymptotically tend to a spatially homogeneous steady state $\boldsymbol{\omega}^{*}=\left(\omega_{1}^{*}, \omega_{2}^{*}, \cdots, \omega_{n}^{*}\right)$, which satisfies

$$
\begin{gathered}
g_{1}\left(\omega_{1}^{*}\right)+f_{1}\left(\omega_{1}^{*}, \omega_{2}^{*}, \cdots, \omega_{n}^{*}\right)=0 \\
g_{2}\left(\omega_{2}^{*}\right)+f_{2}\left(\omega_{1}^{*}, \omega_{2}^{*}, \cdots, \omega_{n}^{*}\right)=0 \\
\vdots \\
g_{n}\left(\omega_{n}^{*}\right)+f_{n}\left(\omega_{1}^{*}, \omega_{2}^{*}, \cdots, \omega_{n}^{*}\right)=0 .
\end{gathered}
$$

We linearize the original nonlinear system (2.1) at the steady state $\boldsymbol{\omega}^{*}$ by letting $\boldsymbol{\omega}(x, t)=$ 
$\boldsymbol{\omega}^{*}+\tilde{\boldsymbol{\omega}}(x, t)$ and drop the ${ }^{\sim}$ for notational convenience. We obtain the linear system

$$
\begin{aligned}
& \frac{\partial \boldsymbol{\omega}(x, t)}{\partial t}=\mathcal{D} \Delta \boldsymbol{\omega}(x, t)+\mathcal{J} \boldsymbol{\omega}(x, t):=\mathcal{L} \boldsymbol{\omega}(x, t), \\
& \frac{\partial \boldsymbol{\omega}(x, t)}{\partial \nu}=0, \quad x \in \Omega
\end{aligned}
$$

where

$$
\boldsymbol{\omega}(x, t)=\left(\begin{array}{c}
\omega_{1}(x, t) \\
\omega_{2}(x, t) \\
\vdots \\
\omega_{n}(x, t)
\end{array}\right), \quad \mathcal{D}=\left(\begin{array}{cccc}
d_{1} & 0 & \cdots & 0 \\
0 & d_{2} & \cdots & 0 \\
\vdots & \vdots & & \vdots \\
0 & 0 & \cdots & d_{n}
\end{array}\right), \quad \mathcal{J}=\left(\begin{array}{cccc}
J_{11} & J_{12} & \cdots & J_{1 n} \\
J_{21} & J_{22} & \cdots & J_{2 n} \\
\vdots & \vdots & & \vdots \\
J_{n 1} & J_{n 2} & \cdots & J_{n n}
\end{array}\right) .
$$

In the above matrix $\mathcal{J}$, we have

$$
\begin{aligned}
& J_{i i}=\left.\left[\frac{d g_{i}\left(\omega_{i}\right)}{d \omega_{i}}+\frac{\partial f_{i}\left(\omega_{1}, \omega_{2}, \cdots, \omega_{n}\right)}{\partial \omega_{i}}\right]\right|_{\omega^{*}}, \quad J_{i j}=\left.\left[\frac{\partial f_{i}\left(\omega_{1}, \omega_{2}, \cdots, \omega_{n}\right)}{\partial \omega_{j}}\right]\right|_{\omega^{*}}, \\
& \text { for } \quad 1 \leq i, j \leq n, \quad i \neq j .
\end{aligned}
$$

Before we define reactivity and amplification envelope, we introduce some additional notation that we will need later in the proofs. In particular, we relate the eigenvalues of (2.3) to the eigenvalues of a simpler problem. Let $0=\mu_{1}<\mu_{2}<\mu_{3}<\cdots$ be the eigenvalues of the operator $-\Delta$ on $\Omega$ which has $\mathcal{C}^{2, \alpha}$ boundary and satisfies homogeneous Neumann boundary condition (Du 2006), i.e.,

$$
\begin{aligned}
& \Delta \phi_{i}(x)+\mu_{i} \phi_{i}(x)=0, \\
& \frac{\partial \phi_{i}(x)}{\partial \nu}=0, x \in \Omega .
\end{aligned}
$$

Let $\mathbf{E}\left(\mu_{i}\right)$ be the eigenspace corresponding to $\mu_{i}$ in $\mathcal{C}^{1}(\bar{\Omega})$ and $\left\{\phi_{i j}: j=1,2, \cdots \operatorname{dim} \mathbf{E}\left(\mu_{i}\right)\right\}$ be an orthogonal basis in $\mathbf{E}\left(\mu_{i}\right)$. Then the function space spanned by $\phi_{i j}$ is invariant under the operator $\mathcal{L}$. Hence, given any $\mu_{i}$, by (2.6), $\lambda_{i}$ is an eigenvalue of (2.3) if and only if it satisfies

$$
\left(-\mu_{i} \mathcal{D}+\mathcal{J}\right) \phi_{i}=\lambda_{i} \phi_{i}
$$

which further leads to the characteristic polynomial

$$
\operatorname{det}\left(\lambda \mathrm{I}+\mu_{i} \mathcal{D}-\mathcal{J}\right)=0 .
$$

Expanding (2.8) gives

$$
\lambda_{i}^{n}+h_{1}\left(\mu_{i}\right) \lambda_{i}^{n-1}+h_{2}\left(\mu_{i}\right) \lambda_{i}^{n-2}+\cdots+h_{n}\left(\mu_{i}\right)=0,
$$

where $h_{j}\left(\mu_{i}\right)$ for $j=1,2, \cdots, n$ are analytical functions of $\mu_{i}$. Because we assume that the steady state $\boldsymbol{\omega}^{*}$ is locally asymptotically stable, we have the condition

$$
\Re\left(\lambda_{1, i}\right)<0, \Re\left(\lambda_{2, i}\right)<0, \cdots, \Re\left(\lambda_{n, i}\right)<0 .
$$


In general, solutions of (2.9) with large $n$ cannot be calculated explicitly but can only be obtained numerically. Therefore, in the analysis below, we use a two-species interaction model as an illustration. This simplification allows us to perform a more detailed analysis but still maintains the essential properties of the general model (2.1). Moreover, the definitions and quantities that we obtain below are not restricted to the two-species interaction model but hold in the general case and can be extended easily. Two-species interactions can be classified, in the simplest case, as cooperative (each species helps the other), competitive (each species inhibits the other), or consumptive (one species benefits, the other suffers). We will see below (Proposition 2.3) that these differences in interactions lead to different upper bounds for the amplification envelope.

\subsection{Reactivity}

Reactivity describes the dynamical behavior of a system immediately after a single small perturbation away from the equilibrium. Extending the ideas by Neubert and Caswell (1997), we define the reactivity in spatial models as

$$
\sigma:=\max _{\left\|\boldsymbol{\omega}_{0}(x)\right\| \neq 0}\left[\left(\frac{1}{\|\boldsymbol{\omega}(x, t)\|} \frac{d\|\boldsymbol{\omega}(x, t)\|}{d t}\right)\right]_{t=0},
$$

where $\boldsymbol{\omega}(x, t)$ is given in $(2.4)$ and $\boldsymbol{\omega}_{0}(x)$ are the initial data of system (2.3). To be consistent with Neubert and Caswell (1997), we take the $L_{2}$ norm in (2.11). A system is said to be reactive if (2.11) is positive, meaning that there exists at least one perturbation that increases before it eventually decays to 0 . To the contrary, a system is non-reactive if (2.11) is negative, which indicates that all perturbations will attenuate initially.

Before we proceed to calculate the reactivity, we first illustrate the definition in (2.11) by using a one dimensional spatial domain $\Omega=(0, l)$. Here and in the following, we denote the inner product in the space $\left(L_{2}(\Omega)\right)^{2}$ by $\langle\cdot, \cdot\rangle$. Because $(2.3)$ is linear, we obtain $\boldsymbol{\omega}(x, t)$ explicitly as $\boldsymbol{\omega}(x, t)=\sum_{k} \boldsymbol{C}_{k} e^{\lambda(k) t} \cos (k x)$, where $k$ is the Fourier mode, $\lambda$ is an eigenvalue dependent on $k$, and $\boldsymbol{C}_{k}=\left(C_{1 k}, C_{2 k}, \cdots, C_{n k}\right)^{\top}$ is a vector of constants determined by initial conditions. By the no-flux boundary conditions, we have $k=(n \pi) / l$. The $L_{2}$ norm of $\boldsymbol{\omega}(x, t)$ can be calculated as $\|\boldsymbol{\omega}(x, t)\|=\sqrt{\left\langle\sum_{k} \boldsymbol{C}_{k} e^{\lambda(k) t} \cos (k x), \sum_{k} \boldsymbol{C}_{k} e^{\lambda(k) t} \cos (k x)\right\rangle}$. From the definition, we see that the $L_{2}$ norm of a vector-valued function $\boldsymbol{\omega}(x, t)$ contains the summation of integrals of functions over all Fourier modes. Therefore, a single Fourier mode may grow such that the solution at a single location increases in magnitude initially, as illustrated in Figure 1(c), even though the $L_{2}$ norm of $\boldsymbol{\omega}(x, t)$ decays (Figure $1(\mathrm{~d}))$.

In non-spatial models, Neubert and Caswell (1997) show that reactivity can be calculated as the largest eigenvalue of a real symmetric matrix. We extend their results and show that the reactivity for infinite dimensional systems is independent of spatial configuration and is the same as the reactivity derived for finite dimensional systems. 
Lemma 2.1. The reactivity in (2.11) can be calculated by the Rayleigh quotient

$$
\sigma=\max _{\left\|\boldsymbol{\omega}_{0}(x)\right\| \neq 0} \frac{\left\langle\boldsymbol{\omega}_{0}, \mathcal{L}_{1} \boldsymbol{\omega}_{0}\right\rangle}{\left\|\boldsymbol{\omega}_{0}(x)\right\|^{2}}
$$

where $\mathcal{L}_{1}=\Delta \mathcal{D}+\left(\mathcal{J}+\mathcal{J}^{\top}\right) / 2$.

Proof. Following (2.11), we have

$$
\begin{aligned}
\frac{d\|\boldsymbol{\omega}(x, t)\|}{d t} & =\frac{d}{d t}\langle\boldsymbol{\omega}, \boldsymbol{\omega}\rangle^{\frac{1}{2}} \\
& =\frac{1}{2}\langle\boldsymbol{\omega}, \boldsymbol{\omega}\rangle^{-\frac{1}{2}}\left[\left\langle\frac{d \boldsymbol{\omega}}{d t}, \boldsymbol{\omega}\right\rangle+\left\langle\boldsymbol{\omega}, \frac{d \boldsymbol{\omega}}{d t}\right\rangle\right] \\
& =\frac{1}{2\|\boldsymbol{\omega}(x, t)\|}[\langle\mathcal{L} \boldsymbol{\omega}, \boldsymbol{\omega}\rangle+\langle\boldsymbol{\omega}, \mathcal{L} \boldsymbol{\omega}\rangle]=\frac{1}{2\|\boldsymbol{\omega}(x, t)\|}\left[\left\langle\boldsymbol{\omega}, \mathcal{L}^{*} \boldsymbol{\omega}\right\rangle+\langle\boldsymbol{\omega}, \mathcal{L} \boldsymbol{\omega}\rangle\right] \\
& =\frac{1}{\|\boldsymbol{\omega}(x, t)\|}\left\langle\boldsymbol{\omega}, \frac{\mathcal{L}^{*}+\mathcal{L}}{2} \boldsymbol{\omega}\right\rangle,
\end{aligned}
$$

where $\mathcal{L}$ is defined in $(2.3)$, and $\mathcal{L}^{*}$ is the adjoint operator of $\mathcal{L}$, defined by $\langle\mathcal{L} \mathbf{u}, \mathbf{v}\rangle=\left\langle\mathbf{u}, \mathcal{L}^{*} \mathbf{v}\right\rangle$. Standard calculations show that the adjoint operator of $\mathcal{L}$ is

$$
\mathcal{L}^{*}=\Delta\left(\begin{array}{cc}
d_{1} & 0 \\
0 & d_{2}
\end{array}\right)+\mathcal{J}^{\top}
$$

Therefore, by combining (2.3) and (2.14), we obtain

$$
\frac{\mathcal{L}^{*}+\mathcal{L}}{2}=\Delta\left(\begin{array}{cc}
d_{1} & 0 \\
0 & d_{2}
\end{array}\right)+\frac{\mathcal{J}+\mathcal{J}^{\top}}{2}=\mathcal{L}_{1}
$$

It is straightforward to verify that the operator $\mathcal{L}_{1}$ above is a self-adjoint operator. By substituting (2.15) back to (2.13) and following the definition in (2.11), we obtain

$$
\sigma=\max _{\left\|\boldsymbol{\omega}_{0}(x)\right\| \neq 0} \frac{\left\langle\boldsymbol{\omega}_{0}, \frac{\mathcal{L}^{*}+\mathcal{L}}{2} \boldsymbol{\omega}_{0}\right\rangle}{\left\|\boldsymbol{\omega}_{0}(x)\right\|^{2}}=\max _{\left\|\boldsymbol{\omega}_{0}(x)\right\| \neq 0} \frac{\left\langle\boldsymbol{\omega}_{0}, \mathcal{L}_{1} \boldsymbol{\omega}_{0}\right\rangle}{\left\|\boldsymbol{\omega}_{0}(x)\right\|^{2}},
$$

which thus completes the proof.

The quantity in (2.16) is the well-known Rayleigh quotient (Strauss 2007). Moreover, since $\mathcal{L}_{1}$ is self-adjoint, it has real eigenvalues. Hence, by Rayleigh's principle, reactivity can be calculated as the largest eigenvalue of the operator $\mathcal{L}_{1}$. The eigenfunction corresponding to the largest eigenvalue maximizes the quantity in (2.16) (Strauss 2007). The largest eigenvalue of the self-adjoint operator exists and can be calculated explicitly in some cases, which leads to the following proposition. 
Proposition 2.1. If a system has only two interacting species, the reactivity in (2.11) can be calculated explicitly as

$$
\sigma=\frac{J_{11}+J_{22}+\sqrt{\left(J_{11}-J_{22}\right)^{2}+\left(J_{12}+J_{21}\right)^{2}}}{2},
$$

where $J_{i j}$ for $1 \leq i, j \leq 2$ are as in $(2.5)$.

Proof. As in Section 2.1, we define $\mu_{i}$ to be the eigenvalues of the Laplacian operator on $\Omega$. Then $\lambda_{i}$ is an eigenvalue of $\mathcal{L}_{1}$ if and only if it is an eigenvalue of the matrix $-\mu_{i} \mathcal{D}+\left(\mathcal{J}+\mathcal{J}^{\top}\right) / 2$. Hence, similar to $(2.7), \lambda_{i}$ is determined by

$$
\left[-\mu_{i}\left(\begin{array}{cc}
d_{1} & 0 \\
0 & d_{2}
\end{array}\right)+\left(\begin{array}{cc}
\bar{J}_{1} & \bar{J}_{2} \\
\bar{J}_{2} & \bar{J}_{3}
\end{array}\right)\right]\left(\begin{array}{l}
u_{1} \\
u_{2}
\end{array}\right)=\lambda_{i}\left(\begin{array}{l}
u_{1} \\
u_{2}
\end{array}\right),
$$

where $\bar{J}_{1}=J_{11}, \bar{J}_{2}=\left(J_{12}+J_{21}\right) / 2, \bar{J}_{3}=J_{22}$ and $\mathbf{u}=\left(u_{1}(x), u_{2}(x)\right)^{\top}$ is the eigenfunction. From (2.18), we obtain the characteristic equation as

$$
\left(\lambda+d_{1} \mu_{i}-\bar{J}_{1}\right)\left(\lambda+d_{2} \mu_{i}-\bar{J}_{3}\right)-\bar{J}_{2}^{2}=0
$$

which has the solutions,

$$
\begin{aligned}
\lambda_{1, i} & =\frac{-\left[\left(d_{1}+d_{2}\right) \mu_{i}-\left(\bar{J}_{1}+\bar{J}_{3}\right)\right]+\sqrt{\delta}}{2}, \lambda_{2, i}=\frac{-\left[\left(d_{1}+d_{2}\right) \mu_{i}-\left(\bar{J}_{1}+\bar{J}_{3}\right)\right]-\sqrt{\delta}}{2}, \\
\delta & =\left[\left(d_{2}-d_{1}\right) \mu_{i}+\left(\bar{J}_{1}-\bar{J}_{3}\right)\right]^{2}+4 \bar{J}_{2}^{2} \geq 0
\end{aligned}
$$

for each $\mu_{i}$. Direct comparison shows that $\lambda_{1, i}-\lambda_{2, i}=\sqrt{\delta}>0$, so that

$$
\lambda_{1, i}>\lambda_{2, i}
$$

Now we compare $\lambda_{1, i}$ for $i=1,2, \cdots$ to find the maximum eigenvalue. Notice that since the domain $\Omega$ is bounded, (2.6) has a discrete set of eigenvalues $\mu_{i}$ for $i=1,2, \cdots$. From (2.20), we have

$$
\frac{d \lambda_{1, i}}{d \mu_{i}}=-\frac{d_{1}+d_{2}}{2}+\frac{\left[\left(d_{2}-d_{1}\right) \mu_{i}+\left(\bar{J}_{1}-\bar{J}_{3}\right)\right]\left(d_{2}-d_{1}\right)}{2 \sqrt{\delta}},
$$

which implies

$$
\frac{d \lambda_{1, i}}{d \mu_{i}}<0 \Leftrightarrow \frac{\left[\left(d_{2}-d_{1}\right) \mu_{i}+\left(\bar{J}_{1}-\bar{J}_{3}\right)\right]\left(d_{2}-d_{1}\right)}{d_{1}+d_{2}}<\sqrt{\delta} .
$$

If $\left(\bar{J}_{1}-\bar{J}_{3}\right)\left(d_{2}-d_{1}\right) \geq 0$, then $(2.23)$ leads to

$$
\begin{aligned}
\frac{d \lambda_{1, i}}{d \mu_{i}}<0 & \Leftrightarrow\left[\frac{\left[\left(d_{2}-d_{1}\right) \mu_{i}+\left(\bar{J}_{1}-\bar{J}_{3}\right)\right]\left(d_{2}-d_{1}\right)}{d_{1}+d_{2}}\right]^{2}<\delta \\
& \Leftrightarrow\left[\left(\frac{d_{2}-d_{1}}{d_{1}+d_{2}}\right)^{2}-1\right]\left[\left(d_{2}-d_{1}\right) \mu_{i}+\bar{J}_{1}-\bar{J}_{3}\right]^{2}<0 \\
& \Leftrightarrow\left(\frac{d_{2}-d_{1}}{d_{1}+d_{2}}\right)^{2}<1 \Leftrightarrow d_{1} d_{2}>0,
\end{aligned}
$$


which is satisfied. If $\left(\bar{J}_{1}-\bar{J}_{3}\right)\left(d_{2}-d_{1}\right)<0$ and $\mu_{i}>-\left(\left(\bar{J}_{1}-\bar{J}_{3}\right)\left(d_{2}-d_{1}\right)\right) /\left(d_{2}-d_{1}\right)^{2}$, then a similar calculation to $(2.24)$ shows that $d \lambda_{1, i} / d \mu_{i}<0$ is satisfied. If $\left(\bar{J}_{1}-\bar{J}_{3}\right)\left(d_{2}-d_{1}\right)<0$ and $\mu_{i}<-\left(\left(\bar{J}_{1}-\bar{J}_{3}\right)\left(d_{2}-d_{1}\right)\right) /\left(d_{2}-d_{1}\right)^{2}$, then it is clear that $d \lambda_{1, i} / d \mu_{i}<0$ is satisfied because the left-hand side of (2.23) is negative.

Therefore, if $d_{1} \neq d_{2}, \lambda_{1, i}$ is a decreasing function of $\mu_{i}$ and is maximized at $i=1$, i.e., $\mu_{1}=0$. For a special case $d_{1}=d_{2}$, directly from (2.22), we obtain that $d \lambda_{1, i} / d \mu_{i}=-\left(d_{1}+d_{2}\right) / 2$. This shows that if $d_{1}=d_{2}, \lambda_{1, i}$ remains as a constant for all $\mu_{i}$ and hence, without loss of generality, we can still choose $\lambda_{1,1}$ as the maximum. In summary,

$$
\max _{i=1,2, \cdots} \lambda_{1, i}:=\lambda_{\max }=\frac{\bar{J}_{1}+\bar{J}_{3}+\sqrt{\left(\bar{J}_{1}-\bar{J}_{3}\right)^{2}+4 \bar{J}_{2}^{2}}}{2},
$$

which completes the proof.

As the proof above shows, the maximal eigenvalue is achieved when $i=1$, i.e., $\mu_{1}=0$. Moreover, the spatial mode $\mu_{1}=0$ has a constant eigenfunction and leads to a reduced nonspatial model. Therefore, the reactivity in (2.17) is exactly the same as the reactivity found in Neubert and Caswell (1997). This shows that for a spatial model where two species interact and move randomly within a habitat, whether the model is reactive or non-reactive is solely determined by its local interaction terms. Random dispersal of species has no impact on the reactivity of the system.

\subsection{Amplification Envelope}

Reactivity measures the behavior of solutions immediately after a perturbation and therefore provides information of a system as $t \rightarrow 0$. On the other hand, asymptotic stability quantifies the long-term behavior of solutions as $t \rightarrow \infty$. To better understand the dynamical behavior for intermediate times, $0<t<\infty$, we now study the amplification envelope, which bridges the gap between reactivity and asymptotic stability of a system.

Following Neubert and Caswell (1997), we define the amplification envelope of the linearized reaction-diffusion system $(2.3)$ as

$$
\rho(t):=\max _{\left\|\boldsymbol{\omega}_{0}(x)\right\| \neq 0} \frac{\|\boldsymbol{\omega}(x, t)\|}{\left\|\boldsymbol{\omega}_{0}(x)\right\|}=\max _{\left\|\boldsymbol{\omega}_{0}(x)\right\|=1}\|\boldsymbol{\omega}(x, t)\| .
$$

From the definition, we see that the amplification envelope measures the maximum of the relative amplification over all possible initial perturbations. For sufficiently large $t$, we have $\|\boldsymbol{\omega}(x, t)\| \rightarrow 0$ because we assume that the steady state is asymptotically stable. Hence, $\rho(t) \rightarrow 0$ as $t \rightarrow \infty$. On the other hand, $\rho(t) \rightarrow 1$ as $t \rightarrow 0$ by continuity. Now we proceed to analyze the profile of 
$\rho(t)$ when $0<t<\infty$. Note that for finite-dimensional models, as shown in Neubert and Caswell (1997), the definition for $\rho(t)$ immediately gives

$$
\rho(t)=\max _{\left\|\boldsymbol{\omega}_{0}\right\| \neq 0} \frac{\|\boldsymbol{\omega}(t)\|}{\left\|\boldsymbol{\omega}_{0}\right\|}=\max _{\left\|\boldsymbol{\omega}_{0}\right\| \neq 0} \frac{\left\|e^{\mathcal{J} t} \boldsymbol{\omega}_{0}\right\|}{\left\|\boldsymbol{\omega}_{0}\right\|}=\left\|e^{\mathcal{J} t}\right\| \|,
$$

where $\||\cdot|||$ denotes the matrix norm. This norm can be calculated numerically by standard methods. However, in the infinite dimensional case, we can only estimate the norm in (2.25) to obtain an upper bound of the profile $\rho(t)$.

We start by obtaining the explicit solution of $\boldsymbol{\omega}(x, t)$. Because system (2.3) is linear, we seek solutions as $\omega_{1}(x, t)=e^{\lambda t} \phi_{1}(x), \omega_{2}(x, t)=e^{\lambda t} \phi_{2}(x)$. Then by plugging in the Ansatz into (2.3), we obtain that

$$
\begin{aligned}
& \lambda \phi_{1}(x)=d_{1} \Delta \phi_{1}(x)+J_{11} \phi_{1}(x)+J_{12} \phi_{2}(x), \\
& \lambda \phi_{2}(x)=d_{2} \Delta \phi_{2}(x)+J_{21} \phi_{1}(x)+J_{22} \phi_{2}(x) .
\end{aligned}
$$

We may assume that $\phi_{1}(x)$ and $\phi_{2}(x)$ both satisfy (2.6) and vary by constants $c_{i}$. We denote as $\mu_{i}$ an eigenvalue of a Laplacian operator $\Delta$ with homogeneous Neumann boundary condition. Then without loss of generality, let $\phi_{1}(x)=\phi_{1, i}(x)=\phi_{i}(x)$ and $\phi_{2}(x)=\phi_{2, i}(x)=c_{i} \phi_{i}(x)$, where $\phi_{i}(x)$ is the eigenfunction of the Laplacian $\Delta$ corresponding to the eigenvalue $\mu_{i}$. Then we substitute $\phi_{1, i}(x)$ and $\phi_{2, i}(x)$ into (2.26) and obtain

$$
\begin{gathered}
\lambda \phi_{i}(x)=-d_{1} \mu_{i} \phi_{i}(x)+J_{11} \phi_{i}(x)+J_{12} c_{i} \phi_{i}(x), \\
\lambda c_{i} \phi_{i}(x)=-d_{2} \mu_{i} c_{i} \phi_{i}(x)+J_{21} \phi_{i}(x)+J_{22} c_{i} \phi_{i}(x),
\end{gathered}
$$

which further gives

$$
\begin{aligned}
& \lambda+d_{1} \mu_{i}-J_{11}-J_{12} c_{i}=0, \\
& \lambda c_{i}+d_{2} \mu_{i} c_{i}-J_{21}-J_{22} c_{i}=0 .
\end{aligned}
$$

If $J_{12} \neq 0$, we eliminate $c_{i}$ from (2.27) and obtain

$$
\left(\lambda+d_{2} \mu_{i}-J_{22}\right)\left(\lambda+d_{1} \mu_{i}-J_{11}\right)-J_{12} J_{21}=0 .
$$

If $J_{12}=0$, then from $(2.27)$, still we are able to find

$$
c_{i}=\frac{J_{21}}{J_{11}-d_{1} \mu_{i}+d_{2} \mu_{i}-J_{22}}
$$

as long as $J_{11}-d_{1} \mu_{i}+d_{2} \mu_{i}-J_{22} \neq 0$. Here, (2.28) is a simplified version of (2.9) and can be written in the equivalent form

$$
\lambda_{i}^{2}+h_{1}\left(\mu_{i}\right) \lambda_{i}+h_{2}\left(\mu_{i}\right)=0
$$

where

$$
\begin{aligned}
& h_{1}\left(\mu_{i}\right)=\left(d_{1}+d_{2}\right) \mu_{i}-\left(J_{11}+J_{22}\right), \\
& h_{2}\left(\mu_{i}\right)=d_{1} d_{2} \mu_{i}^{2}-\left(J_{11} d_{2}+J_{22} d_{1}\right) \mu_{i}+J_{11} J_{22}-J_{12} J_{21} .
\end{aligned}
$$


From (2.29), we obtain two eigenvalues corresponding to each $\mu_{i}$ as

$$
\lambda_{1, i}=\frac{-h_{1}\left(\mu_{i}\right)+\sqrt{h_{1}^{2}\left(\mu_{i}\right)-4 h_{2}\left(\mu_{i}\right)}}{2}, \lambda_{2, i}=\frac{-h_{1}\left(\mu_{i}\right)-\sqrt{h_{1}^{2}\left(\mu_{i}\right)-4 h_{2}\left(\mu_{i}\right)}}{2} .
$$

Therefore, by (2.26) and (2.30), we obtain the general solution of (2.3) as

$$
\begin{aligned}
& \omega_{1}(x, t)=\sum_{i=1}^{\infty}\left(k_{1, i} e^{\lambda_{1, i} t}+k_{2, i} e^{\lambda_{2, i} t}\right) \phi_{i}(x) \\
& \omega_{2}(x, t)=\sum_{i=1}^{\infty}\left(k_{1, i} c_{1, i} e^{\lambda_{1, i} t}+k_{2, i} c_{2, i} e^{\lambda_{2, i} t}\right) \phi_{i}(x)
\end{aligned}
$$

where $k_{1, i}, k_{2, i}$ are constants determined by initial conditions and $c_{1, i}, c_{2, i}$ can be obtained from $(2.27)$ as

$$
c_{1, i}=\frac{\lambda_{1, i}+d_{1} \mu_{i}-J_{11}}{J_{12}}, c_{2, i}=\frac{\lambda_{2, i}+d_{1} \mu_{i}-J_{11}}{J_{12}},
$$

if $J_{12} \neq 0$. In (2.31), because the eigenfunction $\phi_{i}(x)$ for $i \geq 1$ satisfies $(2.6)$, under the $L_{2}$ norm, we are able to normalize $\phi_{i}(x)$ such that

$$
\left\|\phi_{i}(x)\right\|_{L_{2}}=1
$$

Hence, from (2.33), given initial conditions $\omega_{10}(x), \omega_{20}(x)$ of system $(2.31)$, we obtain

$$
k_{1, i}+k_{2, i}=\left\langle\omega_{10}(x), \phi_{i}(x)\right\rangle, k_{1, i} c_{1, i}+k_{2, i} c_{2, i}=\left\langle\omega_{20}(x), \phi_{i}(x)\right\rangle,
$$

which further leads to

$$
k_{1, i}=\frac{1}{c_{1, i}-c_{2, i}}\left\langle-c_{2, i} \omega_{10}(x)+\omega_{20}(x), \phi_{i}(x)\right\rangle, k_{2, i}=\frac{1}{c_{1, i}-c_{2, i}}\left\langle c_{1, i} \omega_{10}(x)-\omega_{20}(x), \phi_{i}(x)\right\rangle .
$$

Here for $\lambda_{1, i}, \lambda_{2, i}$ in $(2.30)$, similar to $(2.10)$, we have

$$
\Re \lambda_{1, i}<0, \quad \Re \lambda_{2, i}<0,
$$

since that the steady state is asymptotically stable.

Now we are in the position to estimate the amplification envelope, $\rho(t)$, given by $(2.25)$ as

$$
\rho(t)=\max _{\left\|\boldsymbol{\omega}_{0}(x)\right\|_{L_{2}}=1} \sqrt{\left\|\omega_{1}(x, t)\right\|^{2}+\left\|\omega_{2}(x, t)\right\|^{2}}
$$

where

$$
\begin{aligned}
& \left\|\omega_{1}(x, t)\right\|^{2}=\sum_{i=1}^{\infty} \overline{\left(k_{1, i} e^{\lambda_{1, i} t}+k_{2, i} e^{\lambda_{2, i} t}\right)}\left(k_{1, i} e^{\lambda_{1, i} t}+k_{2, i} e^{\lambda_{2, i} t}\right), \\
& \left\|\omega_{2}(x, t)\right\|^{2}=\sum_{i=1}^{\infty} \overline{\left(k_{1, i} c_{1, i} e^{\lambda_{1, i} t}+k_{2, i} c_{2, i} e^{\lambda_{2, i} t}\right)}\left(k_{1, i} c_{1, i} e^{\lambda_{1, i} t}+k_{2, i} c_{2, i} e^{\lambda_{2, i} t}\right),
\end{aligned}
$$


and $^{-}$represents the complex conjugate.

First, we analyze a simpler case where for all $i$, with $i=1,2, \cdots$ the condition

$$
f\left(\mu_{i}\right):=h_{1}^{2}\left(\mu_{i}\right)-4 h_{2}\left(\mu_{i}\right)>0
$$

holds. The assumption (2.38) implies that $\lambda_{1, i}$ and $\lambda_{2, i}$ are real eigenvalues and we can choose

$$
\lambda_{1, i}>\lambda_{2, i}
$$

In addition, we reorganize (2.38) such that

$$
f\left(\mu_{i}\right)=A_{1}\left(\mu_{i}\right)^{2}+A_{2} \mu_{i}+A_{3}
$$

where

$$
A_{1}=\left(d_{1}-d_{2}\right)^{2}, \quad A_{2}=2\left(d_{2}-d_{1}\right)\left(J_{11}-J_{22}\right), \quad A_{3}=\left(J_{11}-J_{22}\right)^{2}+4 J_{12} J_{21} .
$$

Since (2.38) is satisfied for all $\mu_{i} \geq 0$, it is obvious that $f\left(\mu_{1}\right)=f(0)>0$, which gives $A_{3}>0$ immediately. Let

$$
\begin{aligned}
f_{1}\left(\mu_{i}\right) & :=\frac{\frac{1}{2}\left|\mu_{i}\left(d_{1}-d_{2}\right)+\left(J_{22}-J_{11}\right)\right|+\left|J_{12} J_{21}\right|}{A_{1} \mu_{i}^{2}+A_{2} \mu_{i}+A_{3}} \\
f_{2}\left(\mu_{i}\right) & :=\frac{\frac{1}{2}\left|\mu_{i}\left(d_{1}-d_{2}\right)+\left(J_{22}-J_{11}\right)\right|+\left|J_{12}\right|^{2}}{A_{1} \mu_{i}^{2}+A_{2} \mu_{i}+A_{3}}
\end{aligned}
$$

We first state the following Lemma.

Lemma 2.2. The maximum of $f_{1}\left(\mu_{i}\right)$ and $f_{2}\left(\mu_{i}\right)$ exists. We denote it as $A_{1}^{*}=\max \left(f_{1}\left(\mu_{i}\right)\right)$ and $A_{2}^{*}=\max \left(f_{2}\left(\mu_{i}\right)\right)$.

Proof. Similar to the proof of Proposition 2.1, we regard $\mu_{i} \geq 0$ as a continuous variable here. Then it is straightforward to obtain

$$
f_{1}(0)=\frac{\frac{1}{2}\left|J_{22}-J_{11}\right|+\left|J_{12} J_{21}\right|}{A_{3}}>0, \quad f_{2}(0)=\frac{\frac{1}{2}\left|J_{22}-J_{11}\right|+\left|J_{12}\right|^{2}}{A_{3}}>0 .
$$

Moreover, we have

$$
\begin{aligned}
\lim _{\mu_{i} \rightarrow \infty} f_{1}\left(\mu_{i}\right) & =\lim _{\mu_{i} \rightarrow \infty} \frac{\frac{1}{2}\left|\left(d_{1}-d_{2}\right)+\left(J_{22}-J_{11}\right) / \mu_{i}\right|+\left|J_{12} J_{21}\right| / \mu_{i}}{A_{1} \mu_{i}+A_{2}+A_{3} / \mu_{i}}=0, \\
\lim _{\mu_{i} \rightarrow \infty} f_{2}\left(\mu_{i}\right) & =\lim _{\mu_{i} \rightarrow \infty}=\frac{\frac{1}{2}\left|\left(d_{1}-d_{2}\right)+\left(J_{22}-J_{11}\right) / \mu_{i}\right|+\left|J_{12}\right|^{2} / \mu_{i}}{A_{1} \mu_{i}+A_{2}+A_{3} / \mu_{i}}=0 .
\end{aligned}
$$

Then by continuity, the upper bounds of $f_{1}\left(\mu_{i}\right), f_{2}\left(\mu_{i}\right)$ exist, denoted by $A_{1}^{*}, A_{2}^{*}$ respectively, which completes the proof. 
By Lemma 2.2, we are now able to obtain the estimate of $\ln \rho(t)$, which is shown in the following Theorem.

Theorem 2.2. The amplification envelope defined in (2.25) is bounded above by

$$
\ln \rho(t) \leq \min \left\{\sigma t, \lambda_{1}^{*} t+\ln \sqrt{2+4\left|J_{12}-J_{21}\right| \max \left\{A_{1}^{*}, A_{2}^{*}\right\}}\right\}
$$

where $\lambda_{1}^{*}$ is the largest eigenvalue among $\lambda_{1, i}$ for $i=1,2, \cdots$ and $\lambda_{1, i}$ are given in $(2.30)$.

The proof of Theorem 2.2 is given in the appendix. Here, we briefly describe the main idea of deriving the estimated upper bound in (2.44). Because the assumptions (2.35) and (2.38) hold, in Theorem 2.2, we know that $\lambda_{1}^{*}$ is real and negative. Then $\rho(t)$ is bounded above by an exponentially decaying function. Accordingly, $\ln \rho(t)$ is bounded above by a linearly decreasing function. Notice that the linearly decreasing function gives a good approximation of $\ln \rho(t)$ when $t$ is large, but lacks accuracy when $t$ is close to 0 . Therefore, to better approximate $\ln \rho(t)$, we derive another upper bound as $\sigma t$, where $\sigma$ is the reactivity defined in (2.11). Hence, the min of the two functions is an upper bound. In Figure 1, the solid line shows the estimated upper bound under one set of parameters.

We should note that because $\rho(t)$ is defined to be the maximum relative growth of all possible perturbations, $\rho(t)$ in fact represents the worst case scenario. The estimate that we obtain in (2.44) is yet another upper bound of $\ln \rho(t)$. Nonetheless, the estimate (2.44) is important for understanding transients in spatial systems: the upper bound is independent of spatial location but it depends on the diffusion coefficients. It sheds light on how a perturbation may evolve and how large the perturbation may grow at all possible spatial locations.

Now, given the upper bound of $\ln \rho(t)$ as shown in (2.44), we further explore the maximum $\lambda_{1}^{*}$ among $\lambda_{1, i}$ for $i=1,2, \cdots$. As shown above, (2.38) holds for all $\mu_{i} \geq 0$, which directly leads to $A_{3}>0$ or equivalently

$$
\left(J_{11}-J_{22}\right)^{2}+4 J_{12} J_{21}>0 .
$$

Moreover, (2.38) requires $-A_{2} /\left(2 A_{1}\right)<0$ or $-A_{2} /\left(2 A_{1}\right)>0, A_{2}^{2}-4 A_{1} A_{3}<0$, which can be further simplified as

$$
\left(d_{2}-d_{1}\right)\left(J_{11}-J_{22}\right)>0
$$

or

$$
\left(d_{2}-d_{1}\right)\left(J_{11}-J_{22}\right)<0 \text { and } J_{12} J_{21}>0 .
$$

Assume (2.38) holds. From (2.30), it is clear that

$$
\lambda_{1, i}<0 \Leftrightarrow \sqrt{h_{1}^{2}\left(\mu_{i}\right)-4 h_{2}\left(\mu_{i}\right)}<h_{1}\left(\mu_{i}\right),
$$


which directly leads to

$$
h_{1}\left(\mu_{i}\right)>0 \text { and } \quad h_{2}\left(\mu_{i}\right)>0 .
$$

Because (2.48) is satisfied for all $\mu_{i} \geq 0$, we obtain a necessary condition by substituting $\mu_{1}=0$ into (2.48) as

$$
J_{11}+J_{22}<0 \quad \text { and } \quad J_{11} J_{22}-J_{12} J_{21}>0 .
$$

Moreover, similar to the above analysis, $h_{2}\left(\mu_{i}\right)>0$ requires

$$
J_{11} d_{2}+J_{22} d_{1}<0
$$

or

$$
J_{11} d_{2}+J_{22} d_{1}>0 \quad \text { and } \quad\left(J_{11} d_{2}-J_{22} d_{1}\right)^{2}+4 d_{1} d_{2} J_{12} J_{21}<0 .
$$

Therefore, if (2.45), (2.49) are satisfied, in addition (2.46) or (2.47) holds, and (2.50) or (2.51) holds, the spatially homogeneous steady state $\boldsymbol{\omega}^{*}$ is locally asymptotically stable for all spatial modes $\mu_{i}$. Now under these conditions, we have the following Proposition, which gives $\lambda_{1}^{*}=$ $\max \left(\lambda_{1, i}\right)$ for $i=1,2, \cdots$.

Proposition 2.3. The largest eigenvalue $\lambda_{1}^{*}$ among $\lambda_{1, i}$ for $i=1,2, \cdots$ is $\lambda_{1,1}$ if the system (2.1) is a cooperative or competitive type model. If the system (2.1) is a consumptive (predator-prey) type model, then $\lambda_{1}^{*}$ might be the intermediate eigenvalue $\lambda_{1, n}$ where $n \geq 2$, if certain conditions are satisfied.

The proof of Proposition 2.3 is given in the appendix.

\section{$3 \quad$ Numerical Simulations}

In this section, we illustrate how the transient dynamics depend on the spatial configuration of the initial perturbation from the (asymptotically stable) steady state. Throughout, we use the predator-prey model (1.1) with Neumann boundary conditions. The linearization that defines the reactivity and the amplification envelope is given by equations (1.2).

The dynamics of the non-spatial system (1.1) (i.e., when $d_{1}=d_{2}=0$ ) are well known (Kot 2001). Particularly, a unique positive steady state, $E\left(\omega_{1}^{*}, \omega_{2}^{*}\right)$, explicitly given by $\omega_{1}^{*}=1 / \theta, \omega_{2}^{*}=$ $r_{1}(1-1 / \theta)$, exists if $\theta>1$, i.e., the predator is sufficiently efficient. Since we imposed noflux boundary conditions, every solution to the non-spatial system also represents a spatially homogeneous solution to the spatial system for positive diffusion coefficients. We shall study the transient behavior of the spatially homogeneous coexistence state $E\left(\omega_{1}^{*}, \omega_{2}^{*}\right)$.

First, we linearize (1.1) about $E\left(\omega_{1}^{*}, \omega_{2}^{*}\right)$ and obtain (1.2), where $J_{11}=-r_{1} / \theta, J_{12}=-1 / \theta$, $J_{21}=r_{1}(\theta-1)$, and $J_{22}=0$. We denote the two eigenvalues of the linearized system as $\lambda_{1}, \lambda_{2}$ 
in the absence of diffusion terms. By direct calculations, we obtain that $\lambda_{1}+\lambda_{2}=-r_{1} / \theta<0$, and $\lambda_{1} \lambda_{2}=\left(r_{1} / \theta\right)(\theta-1)>0$ if $\theta>1$, i.e., if $E\left(\omega_{1}^{*}, \omega_{2}^{*}\right)$ is positive (biologically meaningful). In particular, in the presence of diffusion terms, the spatially homogeneous steady state $E\left(\omega_{1}^{*}, \omega_{2}^{*}\right)$ is asymptotically stable, and all (small) perturbations away from the steady state eventually decay to zero (Kuang 2001).

By substituting the above values for $J_{i j}$ into the formula in (2.17), we calculate the reactivity of system (1.2) as

$$
\sigma=\frac{1}{2}\left(-\frac{r_{1}}{\theta}+\sqrt{\left(-\frac{r_{1}}{\theta}\right)^{2}+\left(-\frac{1}{\theta}+r_{1}(\theta-1)\right)^{2}}\right)>0
$$

by Proposition 2.1. Hence, our predator-prey system is a reactive system, and a perturbation can increase in amplitude before it eventually decays.

Now we illustrate the effect of spatial heterogeneity on the transient behavior for system (1.2). We choose the one-dimensional domain $\Omega=[0,4]$. We choose our spatially heterogeneous perturbations to be localized and vary the degree of localization. More specifically, we choose the initial perturbations from a scaled, one-dimensional Gaussian distribution as

$$
\omega_{i 0}(x, 0)=\beta \exp \left(-\frac{\left(x-x_{0}\right)^{2}}{2 \delta^{2}}\right),
$$

for $i=1,2$, where $x_{0}$ is the center of the perturbation and $\delta$ is the standard deviation. As $\delta$ decreases, the perturbations become increasingly localized. Scaling parameter $\beta$ is chosen so that the norms of all perturbations are equal. We denote different perturbations by $\hat{\boldsymbol{\omega}}_{0}^{j}$ for $j=1,2,3 \cdots$ and the scaled solutions as

$$
\gamma_{j}(t)=\left\|\boldsymbol{\omega}^{j}(x, t)\right\| /\left\|\hat{\boldsymbol{\omega}}_{0}^{j}\right\|
$$

which measure the relative deviation of a perturbation from the steady state at any time $t$. Figure 2(a) shows $\gamma_{j}$ for $j=1,2,3$ and the estimated upper bound obtained in Theorem 2.2. For all the perturbations $\hat{\boldsymbol{\omega}}_{0}^{j}$ for $j=1,2,3$, the initial data $\omega_{10}(x, 0)$ and $\omega_{20}(x, 0)$ are equal and satisfy $(3.1)$ with $\delta=0.1,0.8,1.5$ respectively. We vary $\beta$ accordingly for each perturbation such that $\left\|\omega_{10}(x, 0)\right\|=\left\|\omega_{20}(x, 0)\right\|=0.3$ for $\hat{\boldsymbol{\omega}}_{i}$ with $i=1,2,3$. We clearly see the initial increase in norm near $t=0$ for $\gamma_{2}$ and $\gamma_{3}$. For the parameters chosen in Figure 2 (see Figure caption for details), we calculate the reactivity $\sigma=2.8677$ directly from Proposition 2.1. Moreover, we have $A_{1}^{*}=1.5, A_{2}^{*}=0.55$ as defined in Lemma 2.2 , and the largest eigenvalue $\lambda_{1}^{*}=-1.3820$ among $\lambda_{1, i}$ for $i=1,2, \cdots$. These values give the upper bound of the amplification envelope, $\ln \rho(t)$, as shown in the solid curve in Figure 2(a). While different initial perturbations will produce different solutions, none will exceed the amplification envelope. 
Figure 2(b) shows the different initial data that generate $\gamma_{j}$, as defined above with decreasingly localized distribution. Because initial data of the prey and the predator satisfy the same distribution, only the prey density, $\omega_{10}(x, 0)$, is shown. Figure $2(a)$ shows that the transients decay faster if the initial perturbation is more localized. In fact, in the most localized case, the norm attenuates and does not amplify initially. If one were to observe only the last scenario, one might not guess that the system is reactive. Figure 2(a) also indicates that perturbations may grow into a larger amplitude if the perturbations are more spread out initially. We ran simulations with another family of functions, $\cos (n \pi) / L$ for $n=1,2,3 \cdots$, and observed a similar pattern. Thus, we conjecture that spatially homogeneous perturbations may lead to the largest amplification.

Figure 3 illustrates how diffusion rates of interacting species affect transient response to perturbations. Still, we choose initial data that satisfy (3.1) with different standard deviation $\delta$. We set the range of $\delta$ as $0.1-2$. We then plot the maximum of $\gamma$ as a function of $\delta$. For either small diffusion (dashed line) or large diffusion (dotted line) of species, we observe a similar pattern where amplification of perturbations increases with the larger value of $\delta$. The solid line in Figure 3 shows the maximum of $\rho(t)$ in the corresponding non-spatial model. Figure 3 shows that the maximum of $\gamma$ for both small diffusion and large diffusion approach the $\max (\rho(t))$ as $\delta$ increases. This confirms again our previous conjecture that flatter initial perturbations may produce a larger amplitude. Moreover, Figure 3 also indicates that a larger diffusion coefficient tends to inhibit the growth of perturbations because perturbations attenuate over a larger range of $\delta$. This indicates that the diffusion rates play an important role in determining the transient growth of perturbations. When it comes to ecosystem management in spatial systems, we need to consider the movement behavior of species, not only initial distribution of perturbations. Moreover, the amplification envelope obtained from the non-spatial system often overestimates the amplitude that a perturbation may grow into in a spatial setting.

For the previous figures, we chose identical initial data for the prey and predator density, i.e., $\omega_{10}(x, 0)=\omega_{20}(x, 0)$. Moreover, both initial distributions were centered in the middle of the domain and were thus symmetric. Now we explore how spatial location affects the transient behavior of perturbations. We set the Gaussian of the prey's initial density still centered in the middle of the domain, i.e., $x_{0}=2$, but vary the $x_{0}$ for the predator's initial density from 0 to 4 (Figure 4 ). Parameters are the same as in Figure 2, except $\delta$ and $x_{0}$ for the predator's initial density. In Figure 4, simulations with different $\delta$ show a consistent pattern that $\gamma$ achieves its maximum value when the initial data of predator is identical to prey and is symmetrically distributed in the domain. Biologically, this indicates that perturbations starting from different spatial locations between the prey and the predator may inhibit the transient growth of perturbations. 
Finally, Figure 5 shows the evolution of perturbations where perturbations attenuate initially but increase at some intermediate time. More importantly, it shows that the increase following initial decrease may be large and exceed 1 . This phenomenon can be observed in a wide range of simulations by varying $\delta$ or $x_{0}$ in the Gaussian (3.1). For ecosystem management, this indicates

the possibility that the impact of a single perturbation may attain a peak at some delayed time and we have to monitor the system at a longer course of time.

\section{Discussion and Conclusion}

Our work is motivated by the work of Neubert and Caswell (1997), in which transients were studied for non-spatial ODE systems and the terms "reactivity" and "amplification envelope" were introduced to measure transients. Transient behavior is important in applications because it informs us about short-term dynamics that result, for example, from human management interventions in ecosystems. Transients often run on a time scale which is most relevant to such real-life problems (Hastings 2004; Hastings et al. 2018). Standard asymptotic results cannot provide this information. However, in Neubert and Caswell (1997), spatial aspects are ignored and therefore it is unclear how the spatial distribution of perturbations would affect transient behavior. The only two available studies on transient behavior in spatial systems are based on transformation or discretization and application of the finite-dimensional theory (Anderson et al. 2008; Phillips 2017).

We studied transient behavior of spatially distributed population models by using reactiondiffusion systems, where species interact locally and diffuse randomly within a bounded domain. We defined reactivity, which measures the short-term behavior of a system as $t \rightarrow 0$. Furthermore, we derived the explicit formula to calculate reactivity for reaction-diffusion systems and found that the reactivity of the spatial system equals the reactivity of the corresponding non-spatial system.

This result has clear implications for ecosystem management. First, the maximum increase after a perturbation can be predicted from the non-spatial model, independently of the size or shape of the ecosystem. Secondly, this maximum increase occurs for large-scale (homogeneous) perturbations such as a large fire or large-scale (e.g., aerial) spraying. Localized perturbations will not have the maximal short-term effect.

We also studied the amplification envelope, which describes the time evolution of perturbations imposed near the steady state. Given any spatially homogeneous or heterogeneous perturbations, we found an upper bound for the amplification envelope. The upper bound is determined by the reactivity and the largest eigenvalue of a corresponding linearized system. In particular, this upper bound reflects the geometry of the domain and may therefore differ between the spatial 
and non-spatial system. It may also differ depending on the type of biotic interactions between the species, i.e., whether the system is competitive, cooperative or consumptive.

Moreover, we studied the impact of spatially heterogeneous perturbations on transient dynamics by using numerical simulations. We found that spatial heterogeneity may lower the peak of amplification of perturbations. The amplification envelope defined for non-spatial system may overestimate the maximal increase of perturbations. We also explored how diffusion rates affect transient growth of perturbations and found that a large diffusion rate tends to inhibit the amplification of perturbations. The result is somewhat surprising because a large diffusion rate spreads out a localized perturbation more quickly, and we have previously seen that a less localized perturbation is more likely to produce amplification. As a result, intuitively, a large diffusion rate should facilitate the amplification of perturbations.

We point to a few future questions that arise from our work. Firstly, the current theoretical framework only applies to Neumann boundary conditions. However, other boundary conditions such as Dirichlet or Robin boundary conditions may be of interest in some situations. The main difficulty of working with the aforementioned boundary conditions is that the steady state for the non-spatial model is no longer a spatially homogeneous steady state for the spatial model. Therefore, the linearization of the nonlinear system about the homogeneous steady state cannot be applied. Nonetheless, if we assume that a steady state exists for the spatial system, we can still linearize the system about the steady state and study the reactivity and the amplification envelope accordingly. Secondly, our current results, as well as those in Mari et al. (2017); Neubert and Caswell (1997) and most works on transient dynamics, are based on the assumption that solutions tend to a steady state asymptotically. It would be interesting to study transient dynamics when solutions oscillate periodically in the long-time. A first step in this direction in a seasonally forced system was taken by Vesipa and Ridolfi (2017), but spatial aspects were not considered. Thirdly, we only considered the case where populations move randomly in a habitat. However, populations might perceive environmental cues and move directionally, e.g., towards or away from perturbations. Such directional movement may lead to new transient behavior and require a detailed analysis.

\section{Acknowledgement}

XW would like to thank Fields Institute for the Fields-Ontario postdoc fellowship. This work was partially finished while XW and ME attended the thematic program "Emerging Challenges in Mathematical Biology" at Fields Institute. FL is grateful for funding from the NSERC of Canada (RGPIN-2016-04759 and RGPAS-2016-492872). We thank the editor and two anonymous reviewers for their comments, which lead to a substantial improvement of the manuscript. 


\section{References}

Anderson, K. E., Nisbet, R. M., and McCauley, E. (2008). Transient responses to spatial perturbations in advective systems. Bulletin of Mathematical Biology, 70:1480-1502.

Arnoldi, J. F., Loreau, M., and Haegeman, B. (2016). Resilience, reactivity and variability: A mathematical comparison of ecological stability measures. Journal of Theoretical Biology, 389:47-59.

Bailey, H. and Secor, D. H. (2016). Coastal evacuations by fish during extreme weather events. Scientific Reports, 6:30280.

Carpenter, S. R. and Brock, W. A. (2004). Spatial complexity, resilience and policy diversity: fishing on lake-rich landscapes. Ecology and Society, 9:8.

Caswell, H. and Neubert, M. G. (2005). Reactivity and transient dynamics of discrete-time ecological systems. Journal of Difference Equations and Applications, 11:295-310.

Du, Y. (2006). Order Structure and Topological Methods in Nonlinear Partial Differential Equations Vol 1: Maximum Principles and Applications. World Scientific.

Hastings, A. (2004). Transients: the key to long-term ecological understanding? Trends in Ecology and Evolution, 19:39-45.

Hastings, A., Abbott, K. C., Cuddington, K., Francis, T., Gellner, G., Lai, Y. C., Morozov, A., Petrovskii, S., Scranton, K., and Zeeman, M. L. (2018). Transient phenomena in ecology. Science, 361(6406):eaat6412.

Kot, M. (2001). Elements of Mathematical Ecology. Cambridge University Press.

Kuang, Y. (2001). Global stability and persistence in diffusive food chains. ANZIAM Journal, $43: 247-268$.

Mari, L., Casagrandi, R., Rinaldo, A., and Gatto, M. (2017). A generalized definition of reactivity for ecological systems and the problem of transient species dynamics. Methods in Ecology and Evolution, 8:1574-1584.

Matthews, W. J. (1986). Fish faunal structure in an ozark stream: stability, persistence and a catastrophic flood. Copeia, 2:388-397.

Neubert, M. G. and Caswell, H. (1997). Alternatives to resilience for measuring the responses of ecological systems to perturbations. Ecology, 78:653-665. 
Neubert, M. G., Caswell, H., and Murray, J. D. (2002). Transient dynamics and pattern formation: reactivity is necessary for turing instabilities. Mathematical Biosciences, 175:1-11.

Phillips, A. J. (2017). Spatial models as powerful tools for climate change ecology. Electronic Thesis and Dissertation Repository. http://hdl.handle.net/1773/38681.

Pimm, S. L. and Lawton, J. H. (1977). Number of trophic levels in ecological communities. Nature, 268:329-331.

Snyder, R. E. (2010). What makes ecological systems reactive? Theoretical Population Biology, $77: 243-249$.

Stott, I. (2016). Perturbation analysis of transient population dynamics using matrix projection models. Methods in Ecology and Evolution, 7:666-678.

Stott, I., Townley, S., and Hodgson, D. J. (2011). A framework for studying transient dynamics of population projection matrix models. Ecology Letters, 14:959-970.

Strauss, W. A. (2007). Partial Differential Equations: An Introduction, Second Edition. WILEY.

Townley, S., Carslake, D., Kellie-smith, O., Mccarthy, D., and Hodgson, D. (2007). Predicting transient amplification in perturbed ecological systems. Journal of Applied Ecology, 44:12431251.

Verdy, A. and Caswell, H. (2008). Sensitivity analysis of reactive ecological dynamics. Bulletin of Mathematical Biology, 70:1634-1659.

Vesipa, R. and Ridolfi, L. (2017). Impact of seasonal forcing on reactive ecological systems. Journal of Theoretical Biology, 419:23-35.

Wang, H., Zhang, J., and Yang, X. S. (2017). The maximum amplification of perturbations in ecological systems. International Journal of Biomathematics, 10:1750009.

\section{Appendices}

\section{A Proof of Theorem 2.2}

\section{A.1 Proof when (2.38) holds}

In the appendix, we first show the proof of Theorem 2.2, where the estimated upper bound of $\ln \rho(t)$ is given. 
Proof. Because the steady state $\boldsymbol{\omega}^{*}$ remains asymptotically stable, from (2.35) and (2.38), we immediately have that $\lambda_{1, i}, \lambda_{2, i}$ are real eigenvalues and

$$
\lambda_{2, i}<\lambda_{1, i}<0 \quad \text { for } \quad i=1,2, \cdots
$$

Then (2.37) can be further simplified as

$$
\left\|\omega_{1}(x, t)\right\|^{2}=\sum_{i=1}^{\infty}\left|k_{1, i} e^{\lambda_{1, i} t}+k_{2, i} e^{\lambda_{2, i} t}\right|^{2}, \quad\left\|\omega_{2}(x, t)\right\|^{2}=\sum_{i=1}^{\infty}\left|k_{1, i} c_{1, i} e^{\lambda_{1, i} t}+k_{2, i} c_{2, i} e^{\lambda_{2, i} t}\right|^{2}
$$

To estimate the profile $\rho(t)$ from $(2.36)$ is equivalent to maximize

$$
\begin{aligned}
& \left\|\omega_{1}(x, t)\right\|^{2}+\left\|\omega_{2}(x, t)\right\|^{2} \\
& =\sum_{i=1}^{\infty}\left|k_{1, i} e^{\lambda_{1, i} t}+k_{2, i} e^{\lambda_{2, i} t}\right|^{2}+\sum_{i=1}^{\infty}\left|k_{1, i} c_{1, i} e^{\lambda_{1, i} t}+k_{2, i} c_{2, i} e^{\lambda_{2, i} t}\right|^{2}
\end{aligned}
$$

subject to the constrain

$$
\sum_{i=1}^{\infty}\left(k_{1, i}+k_{2, i}\right)^{2}+\sum_{i=1}^{\infty}\left(k_{1, i} c_{1, i}+k_{2, i} c_{2, i}\right)^{2}=1
$$

Because of the infinite sum in (A.3) and (A.4), it is almost impossible to find the exact maximum value of (A.3). Instead, we can find an upper bound of (A.3) subject to (A.4). To simplify notations, we let

$$
M_{1, i}=\int_{\Omega} \omega_{10}(x) \phi_{i}(x) d x, \quad M_{2, i}=\int_{\Omega} \omega_{20}(x) \phi_{i}(x) d x .
$$

Then the constrain (A.4) is equivalent to

$$
\sum_{i=1}^{\infty}\left(M_{1, i}^{2}+M_{2, i}^{2}\right)=1
$$

Reorganizing (A.3) and using (2.39), we obtain

$$
\begin{aligned}
& \left\|\omega_{1}(x, t)\right\|^{2}+\left\|\omega_{2}(x, t)\right\|^{2} \\
& =\sum_{i=1}^{\infty}\left[\left(k_{1, i}+k_{2, i}\right)^{2}\left(e^{2 \lambda_{1, i} t}+e^{2 \lambda_{2, i} t}\right)-\left(k_{1, i} e^{\lambda_{2, i} t}-k_{2, i} e^{\lambda_{1, i} t}\right)^{2}-2 k_{1, i} k_{2, i}\left(e^{2 \lambda_{1, i} t}+e^{2 \lambda_{2, i} t}\right)\right] \\
& +\sum_{i=1}^{\infty}\left[\left(k_{1, i} c_{1, i}+k_{2, i} c_{2, i}\right)^{2}\left(e^{2 \lambda_{1, i} t}+e^{2 \lambda_{2, i} t}\right)-\left(k_{1, i} c_{1, i} e^{\lambda_{2, i} t}-k_{2, i} c_{2, i} e^{\lambda_{1, i} t}\right)^{2}\right. \\
& \left.-2 k_{1, i} k_{2, i} c_{1, i} c_{2, i}\left(e^{2 \lambda_{1, i} t}+e^{2 \lambda_{2, i} t}\right)\right] \\
& \leq \sum_{i=1}^{\infty}\left\{\left[\left(k_{1, i}+k_{2, i}\right)^{2}+\left(k_{1, i} c_{1, i}+k_{2, i} c_{2, i}\right)^{2}\right]\left(e^{2 \lambda_{1, i} t}+e^{2 \lambda_{2, i} t}\right)\right\}
\end{aligned}
$$




$$
\begin{aligned}
& -2 \sum_{i=1}^{\infty}\left(1+c_{1, i} c_{2, i}\right) k_{1, i} k_{2, i}\left(e^{2 \lambda_{1, i} t}+e^{2 \lambda_{2, i} t}\right) \\
& \leq 2 \sum_{i=1}^{\infty}\left[\left(k_{1, i}+k_{2, i}\right)^{2}+\left(k_{1, i} c_{1, i}+k_{2, i} c_{2, i}\right)^{2}\right] e^{2 \lambda_{1, i} t}-2 \sum_{i=1}^{\infty}\left(1+c_{1, i} c_{2, i}\right) k_{1, i} k_{2, i}\left(e^{2 \lambda_{1, i} t}+e^{2 \lambda_{2, i} t}\right) . \\
& \leq 2 e^{2 \lambda_{1}^{*} t} \sum_{i=1}^{\infty}\left(M_{1, i}^{2}+M_{2, i}^{2}\right)+2\left|\sum_{i=1}^{\infty}\left(1+c_{1, i} c_{2, i}\right) k_{1, i} k_{2, i}\left(e^{2 \lambda_{1, i} t}+e^{2 \lambda_{2, i} t}\right)\right| \\
& \leq 2 e^{2 \lambda_{1}^{*} t}+4 \sum_{i=1}^{\infty}\left|\left(1+c_{1, i} c_{2, i}\right) k_{1, i} k_{2, i}\right| e^{2 \lambda_{1, i} t} \leq 2 e^{2 \lambda_{1}^{*} t}+4 e^{2 \lambda_{1}^{*} t} \sum_{i=1}^{\infty}\left|\left(1+c_{1, i} c_{2, i}\right) k_{1, i} k_{2, i}\right|,
\end{aligned}
$$

where $\lambda_{1, i} \leq \lambda_{1}^{*}$ for $i=1,2, \cdots$

Direct calculations show that

$$
1+c_{1, i} c_{2, i}=\frac{J_{12}-J_{21}}{J_{12}}
$$

by using (2.32). It follows that

$$
\begin{aligned}
& \sum_{i=1}^{\infty}\left|\left(1+c_{1, i} c_{2, i}\right) k_{1, i} k_{2, i}\right|=\frac{\left|J_{12}-J_{21}\right|}{\left|J_{12}\right|} \sum_{i=1}^{\infty}\left|k_{1, i} k_{2, i}\right| \\
& =\left|J_{12}\left(J_{12}-J_{21}\right)\right| \sum_{i=1}^{\infty}\left|\frac{\left(-c_{2, i} M_{1, i}+M_{2, i}\right)\left(c_{1, i} M_{1, i}-M_{2, i}\right)}{A_{1} \mu_{i}^{2}+A_{2} \mu_{i}+A_{3}}\right| \\
& =\left|J_{12}-J_{21}\right| \sum_{i=1}^{\infty} \frac{\left|M_{1, i} M_{2, i}\left[\mu_{i}\left(d_{1}-d_{2}\right)+\left(J_{22}-J_{11}\right)\right]-J_{12}^{2} M_{2, i}^{2}+J_{12} J_{21} M_{1, i}^{2}\right|}{A_{1} \mu_{i}^{2}+A_{2} \mu_{i}+A_{3}} \\
& \leq\left|J_{12}-J_{21}\right| \sum_{i=1}^{\infty} \frac{\frac{1}{2}\left|\mu_{i}\left(d_{1}-d_{2}\right)+\left(J_{22}-J_{11}\right)\right|\left(\left|M_{1, i}\right|^{2}+\left|M_{2, i}\right|^{2}\right)+\left|J_{12}^{2}\right| M_{2, i}^{2}+\left|J_{12} J_{21}\right| M_{1, i}^{2}}{A_{1} \mu_{i}^{2}+A_{2} \mu_{i}+A_{3}} \\
& =\left|J_{12}-J_{21}\right|\left(\sum_{i=1}^{\infty} f_{1}\left(\mu_{i}\right) M_{1, i}^{2}+\sum_{i=1}^{\infty} f_{2}\left(\mu_{i}\right) M_{2, i}^{2}\right)
\end{aligned}
$$

where $f_{1}\left(\mu_{i}\right), f_{2}\left(\mu_{i}\right)$ are given in (2.42) and (2.43) respectively. By Lemma 2.2, we have $f_{1}\left(\mu_{i}\right) \leq$ $A_{1}^{*}$ and $f_{2}\left(\mu_{i}\right) \leq A_{2}^{*}$ and $A_{1}^{*}>0, A_{2}^{*}>0$. Then we continue the estimate of (A.7) by using the above inequalities as

$$
\begin{aligned}
& \left|J_{12}-J_{21}\right|\left(\sum_{i=1}^{\infty} f_{1}\left(\mu_{i}\right) M_{1, i}^{2}+\sum_{i=1}^{\infty} f_{2}\left(\mu_{i}\right) M_{2, i}^{2}\right) \leq\left|J_{12}-J_{21}\right|\left(A_{1}^{*} \sum_{i=1}^{\infty} M_{1, i}^{2}+A_{2}^{*} \sum_{i=1}^{\infty} M_{2, i}^{2}\right) \\
& \leq\left|J_{12}-J_{21}\right| \max \left\{A_{1}^{*}, A_{2}^{*}\right\} \sum_{i=1}^{\infty}\left(M_{1, i}^{2}+M_{2, i}^{2}\right)=\left|J_{12}-J_{21}\right| \max \left\{A_{1}^{*}, A_{2}^{*}\right\},
\end{aligned}
$$

where the last equality comes from (A.5). Substituting (A.8) back to (A.6) gives

$$
\rho(t) \leq e^{\lambda_{1}^{*} t} \sqrt{2+4\left|J_{12}-J_{21}\right| \max \left\{A_{1}^{*}, A_{2}^{*}\right\}} .
$$


We take ln for (A.9) at both sides of the inequality and obtain

$$
\ln \rho(t) \leq \lambda_{1}^{*} t+\ln \sqrt{2+4\left|J_{12}-J_{21}\right| \max \left\{A_{1}^{*}, A_{2}^{*}\right\}}
$$

Notice that the above estimate lacks accuracy for $\ln \rho(t)$ when $t \rightarrow 0$ because $\lambda_{1}^{*} t$ decreases linearly while perturbations may grow initially. Hence we give another estimate for $\ln \rho(t)$ when $t$ is close to 0 .

We show that $\ln \rho(t) \leq \sigma t$ for $t \geq 0$, where $\sigma$ is the reactivity defined in (2.11). Suppose not, then there exists at least a $t_{1}>0$ such that $\ln \rho\left(t_{1}\right)>\sigma t_{1}$. Moreover, we have $\ln \rho\left(t_{0}\right)-\sigma t_{0}=0$ if $t_{0}=0$. This leads to

$$
\left.\frac{d \ln \rho(t)}{d t}\right|_{t=t_{1}}>\left.\frac{d(\sigma t)}{d t}\right|_{t=t_{1}}
$$

which directly gives

$$
\left.\frac{1}{\rho\left(t_{1}\right)} \frac{d \rho(t)}{d t}\right|_{t=t_{1}}>\sigma
$$

By the definition of $\rho(t)$ in (2.25), rearranging (A.11) leads to

$$
\left.\max _{\left\|\boldsymbol{\omega}_{0}(x)\right\|=1}\left(\frac{1}{\|\boldsymbol{\omega}(x, t)\|} \frac{d\|\boldsymbol{\omega}(x, t)\|}{d t}\right)\right|_{t=t_{1}}>\sigma .
$$

This contradicts the definition of $\sigma$ in (2.11) because $\sigma$ is chosen as the maximum and $t=0$ can be chosen arbitrarily. Therefore, when $t$ is close to $0, \ln \rho(t)$ is bounded above by $\sigma t$. This together with (A.10) gives (2.44) and thus completes the proof.

\section{A.2 Proof when (2.38) is relaxed}

Now we relax assumption (2.38) such that for some index $j, h_{1}^{2}\left(\mu_{j}\right)-4 h_{2}\left(\mu_{j}\right)<0$ holds. It follows that for these $\mu_{j}$, we have

$$
\lambda_{1, j}=a_{1}\left(\mu_{j}\right)+\mathrm{i} b_{1}\left(\mu_{j}\right), \quad \lambda_{2, j}=a_{1}\left(\mu_{j}\right)-\mathrm{i} b_{1}\left(\mu_{j}\right)
$$

where

$$
a_{1}\left(\mu_{j}\right)=\frac{-h_{1}\left(\mu_{j}\right)}{2}, \quad b_{1}\left(\mu_{j}\right)=\frac{\sqrt{4 h_{2}\left(\mu_{j}\right)-h_{1}^{2}\left(\mu_{j}\right)}}{2} .
$$

Following (2.37), we have

$$
\begin{aligned}
\left\|\omega_{1}(x, t)\right\|^{2} & =\sum_{\substack{i=1 \\
i \neq j}}^{\infty}\left(k_{1, i} e^{\lambda_{1, i} t}+k_{2, i} e^{\lambda_{2, i} t}\right)^{2}+\sum_{j \neq i} \overline{\left(k_{1, j} e^{\lambda_{1, j} t}+k_{2, j} e^{\lambda_{2, j} t}\right)}\left(k_{1, j} e^{\lambda_{1, j} t}+k_{2, j} e^{\lambda_{2, j} t}\right) \\
\left\|\omega_{2}(x, t)\right\|^{2} & =\sum_{\substack{i=1 \\
i \neq j}}^{\infty}\left(k_{1, i} c_{1, i} e^{\lambda_{1, i} t}+k_{2, i} c_{2, i} e^{\lambda_{2, i} t}\right)^{2} \\
& +\sum_{j \neq i} \overline{\left(k_{1, j} c_{1, j} e^{\lambda_{1, j} t}+k_{2, j} c_{2, j} e^{\lambda_{2, j} t}\right)}\left(k_{1, j} c_{1, j} e^{\lambda_{1, j} t}+k_{2, j} c_{2, j} e^{\lambda_{2, j} t}\right) .
\end{aligned}
$$


In the above (A.13), we have

$$
\begin{aligned}
& k_{1, j} e^{\lambda_{1, j} t}+k_{2, j} e^{\lambda_{2, j} t}=\frac{J_{12}}{\lambda_{1, j}-\lambda_{2, j}}\left\langle-\frac{\lambda_{2, j}+d_{1} \mu_{j}-J_{11}}{J_{12}} \omega_{10}(x)+\omega_{20}(x), \phi_{j}(x)\right\rangle e^{\lambda_{1, j} t} \\
& +\frac{J_{12}}{\lambda_{1, j}-\lambda_{2, j}}\left\langle\frac{\lambda_{1, j}+d_{1} \mu_{j}-J_{11}}{J_{12}} \omega_{10}(x)-\omega_{20}(x), \phi_{j}(x)\right\rangle e^{\lambda_{2, j} t} .
\end{aligned}
$$

To simplify the inner product in (A.14), let

$$
f_{1}(x)=\frac{-a_{1}\left(\mu_{j}\right)-d_{1} \mu_{j}+J_{11}}{J_{12}} \omega_{10}(x)+\omega_{20}(x), \quad f_{2}(x)=\frac{b_{1}\left(\mu_{j}\right)}{J_{12}} \omega_{10}(x) .
$$

Then by (A.12) and (A.15), we have

$$
\begin{aligned}
& \left\langle-\frac{\lambda_{2, j}+d_{1} \mu_{j}-J_{11}}{J_{12}} \omega_{10}(x)+\omega_{20}(x), \phi_{j}(x)\right\rangle e^{\lambda_{1, j} t}=e^{a_{1}\left(\mu_{j}\right) t}\left\langle\left(f_{1}(x)+f_{2}(x) \mathrm{i}\right) e^{b_{1}\left(\mu_{j}\right) \mathrm{ti}}, \phi_{j}(x)\right\rangle \\
& =e^{a_{1}\left(\mu_{j}\right) t}\left\langle f_{3}(x)+f_{4}(x) \mathrm{i}, \phi_{j}(x)\right\rangle,
\end{aligned}
$$

where

$$
f_{3}(x)=f_{1}(x) \cos \left(b_{1}\left(\mu_{j}\right) t\right)-f_{2}(x) \sin \left(b_{1}\left(\mu_{j}\right) t\right), \quad f_{4}(x)=f_{1}(x) \sin \left(b_{1}\left(\mu_{j}\right) t\right)+f_{2}(x) \cos \left(b_{1}\left(\mu_{j}\right) t\right) .
$$

Similar calculations show that

$$
\left\langle\frac{\lambda_{1, j}+d_{1} \mu_{j}-J_{11}}{J_{12}} \omega_{10}(x)-\omega_{20}(x), \phi_{j}(x)\right\rangle e^{\lambda_{2, j} t}=e^{a_{1}\left(\mu_{j}\right) t}\left\langle-f_{3}(x)+f_{4}(x) \mathrm{i}, \phi_{j}(x)\right\rangle .
$$

Furthermore, by (A.12), it is obvious that $\lambda_{1, j}-\lambda_{2, j}=2 b_{1}\left(\mu_{j}\right)$ i. Then substituting (A.16) and (A.17) back to (A.14) gives

$$
k_{1, j} e^{\lambda_{1, j} t}+k_{2, j} e^{\lambda_{2, j} t}=\frac{J_{12} e^{a_{1}\left(\mu_{j}\right) t}}{2 b_{1}\left(\mu_{j}\right)}\left\langle 2 f_{4}(x), \phi_{j}(x)\right\rangle,
$$

which is a real number. Hence, we obtain

$$
k_{1, j} e^{\lambda_{1, j} t}+k_{2, j} e^{\lambda_{2, j} t}=\overline{k_{1, j} e^{\lambda_{1, j} t}+k_{2, j} e^{\lambda_{2, j} t}}
$$

which further gives

$$
\begin{aligned}
\left\|\omega_{1}(x, t)\right\|^{2} & =\sum_{\substack{i=1 \\
i \neq j}}^{\infty}\left(k_{1, i} e^{\lambda_{1, i} t}+k_{2, i} e^{\lambda_{2, i} t}\right)^{2}+\sum_{j \neq i} \overline{\left(k_{1, j} e^{\lambda_{1, j} t}+k_{2, j} e^{\lambda_{2, j} t}\right)}\left(k_{1, j} e^{\lambda_{1, j} t}+k_{2, j} e^{\lambda_{2, j} t}\right) \\
& =\sum_{i=1}^{\infty}\left(k_{1, i} e^{\lambda_{1, i} t}+k_{2, i} e^{\lambda_{2, i} t}\right)^{2} .
\end{aligned}
$$

Similar calculations show that $\left\|\omega_{2}(x, t)\right\|^{2}$ from (A.13) can be reduced to the equivalent form in (A.2). Therefore, as long as (2.35) is satisfied, even if some eigenvalues $\lambda_{1, j}, \lambda_{2, j}$ have imaginary parts, expressions in (A.3) and (A.4) remain the same, which leads to similar results for the upper bound of the amplification envelope $\rho(t)$. 


\section{B Proof of Proposition 2.3}

The following gives the proof of Proposition 2.3.

Proof. Assume that (2.45), (2.47), (2.49) and (2.50) are satisfied. Direct calculations give

$$
\begin{aligned}
\frac{d \lambda_{1, i}}{d \mu_{i}} & =\frac{\left[\left(d_{1}-d_{2}\right)^{2} \mu_{i}+\left(d_{2}-d_{1}\right)\left(J_{11}-J_{22}\right)-\left(d_{1}+d_{2}\right) \sqrt{h_{1}^{2}\left(\mu_{i}\right)-4 h_{2}\left(\mu_{i}\right)}\right]}{2 \sqrt{h_{1}^{2}\left(\mu_{i}\right)-4 h_{2}\left(\mu_{i}\right)}} \\
& :=\frac{1}{2 \sqrt{h_{1}^{2}\left(\mu_{i}\right)-4 h_{2}\left(\mu_{i}\right)}} g\left(\mu_{i}\right) .
\end{aligned}
$$

From (B.1), it is clear that

$$
\frac{d \lambda_{1, i}}{d \mu_{i}}<0 \Leftrightarrow g\left(\mu_{i}\right)<0 .
$$

Let $\bar{\mu}=-\left(J_{11}-J_{22}\right) /\left(d_{2}-d_{1}\right)>0$, by $(2.47)$. If $\mu_{i}<\bar{\mu}$, then $g\left(\mu_{i}\right)<0$ is satisfied. If $\mu_{i}>\bar{\mu}$, $g\left(\mu_{i}\right)<0$ leads to

$$
a_{1}\left(\mu_{i}\right)^{2}+a_{2}\left(\mu_{i}\right)+a_{3}>0
$$

where

$$
\begin{aligned}
& a_{1}=4 d_{1} d_{2}\left(d_{1}-d_{2}\right)^{2}, \quad a_{2}=8 d_{1} d_{2}\left(d_{2}-d_{1}\right)\left(J_{11}-J_{22}\right), \\
& a_{3}=4\left\{J_{12} J_{21}\left(d_{1}^{2}+d_{2}^{2}\right)+d_{1} d_{2}\left[2 J_{12} J_{21}+\left(J_{11}-J_{22}\right)^{2}\right]\right\}, \\
& a_{2}^{2}-4 a_{1} a_{3}=-64 d_{1} d_{2}\left(d_{1}-d_{2}\right)^{2} J_{12} J_{21}\left(d_{1}+d_{2}\right)^{2} .
\end{aligned}
$$

From (B.4), it is clear that $a_{1}>0, a_{2}<0, a_{3}>0$ and $a_{2}^{2}-4 a_{1} a_{3}<0$ when (2.45), (2.47), (2.49) and (2.50) hold. This shows that (B.3) is satisfied for all $\mu_{i} \geq 0$, which further leads to $\left(d \lambda_{1, i}\right) /\left(d \mu_{i}\right)<0$.

Therefore, $\lambda_{1, i}$ is a decreasing function of $\mu_{i}$ and is maximized at $\lambda_{1,1}$, i.e. $\mu_{i}=0$, which in fact agrees with the largest eigenvalue corresponding to the spatial mode zero. We notice that the condition $J_{12} J_{21}>0$ in (2.47) implies that the system describes competitive or cooperative interactions. This suggests that for such type of systems, the upper bound of the amplification envelope is, in fact, regulated by the largest eigenvalue of the non-spatial model.

Now assume that (2.45), (2.46), (2.49) and (2.51) are satisfied. Following similar calculations as (B.1), we obtain the same (B.2), (B.3) and (B.4). Condition (2.51) directly gives $J_{12} J_{21}<0$, which further leads to $a_{1}>0, a_{2}>0$ and $a_{2}^{2}-4 a_{1} a_{3}>0$ but the sign of $a_{3}$ is undetermined.

If $a_{3}>0$, then (B.3) holds for all $\mu_{i} \geq 0$. This again shows that $\lambda_{1, i}$ is a decreasing function of $\mu_{i}$ and is maximized at $\lambda_{1,1}$. If $a_{3}<0$, a unique positive root of $a_{1}\left(\mu_{i}\right)^{2}+a_{2}\left(\mu_{i}\right)+a_{3}=0$ exists and we denote the root by $\bar{\mu}_{1}$. Direct calculations show that

$$
\bar{\mu}_{1}=\frac{-a_{2}+\sqrt{a_{2}^{2}-4 a_{1} a_{3}}}{2 a_{1}} .
$$


Following (B.5), if $\mu_{i}<\bar{\mu}_{1}$, then (B.3) is violated, which further leads to $d \lambda_{1, i} / d \mu_{i}>0$. If $\mu_{i}>\bar{\mu}_{1}$, then (B.3) holds, which gives $d \lambda_{1, i} / d \mu_{i}<0$. Hence, by the above discussion, $\mu_{i}=\bar{\mu}_{1}$ is a local maximum and maximizes $\lambda_{1, i}$ at some intermediate $i$. Still in the analysis, $\mu_{i}$ is regarded as a continuous variable but $\bar{\mu}_{1}$ provides an approximation for the $\mu_{i}$ which maximizes $\lambda_{1, i}$. In fact, when $\mu_{i}$ is discrete, it is straightforward to choose $\mu_{j}$ and $\mu_{j+1}$ which satisfy

$$
\mu_{j}<\bar{\mu}_{1}<\mu_{j+1}
$$

and compare $\lambda_{1, j}, \lambda_{1, j+1}$ to find $\lambda_{1}^{*}=\max \left\{\lambda_{1, j}, \lambda_{1, j+1}\right\}$.

We notice that $J_{12} J_{21}<0$ above indicates that the system is a predator-prey type model. Different from the previous case where $\lambda_{1}^{*}=\lambda_{1,1}$ if the system is a competitive or cooperative type, the upper bound of $\rho(t)$ for a predator-prey type system needs not to be the largest eigenvalue corresponding to spatial mode 0 but may be regulated by the largest eigenvalue corresponding to some intermediate spatial mode.

We finally discuss the case where (2.45), (2.46), (2.49) and (2.50) hold. Following the same steps in the previous analysis, we obtain the same (B.3) if $d \lambda_{1, i} / d \mu_{i}<0$. By the assumptions (2.45), (2.46), (2.49) and (2.50), we have $a_{1}>0, a_{2}>0$ but the signs of $a_{3}$ and $a_{2}^{2}-4 a_{1} a_{3}$ are undetermined. Still, it is straight forward that $a_{2}^{2}-4 a_{1} a_{3}>0$ if and only if $J_{12} J_{21}<0$. If $a_{3}>0$, then (B.3) is satisfied regardless of the sign of $a_{2}^{2}-4 a_{1} a_{3}$. This implies that $\lambda_{1, i}$ is a decreasing function of $\mu_{i}$ and is maximized at $\lambda_{1,1}$. If $a_{3}<0$, we have $a_{2}^{2}-4 a_{1} a_{3}>0$ and therefore $J_{12} J_{21}<0$ as a necessary condition. This results in a similar case to the above one, where $\lambda_{1, i}$ is maximized at some intermediate $\lambda_{1, n}$ for $n \geq 2$. The analysis is similar and is thus omitted. 


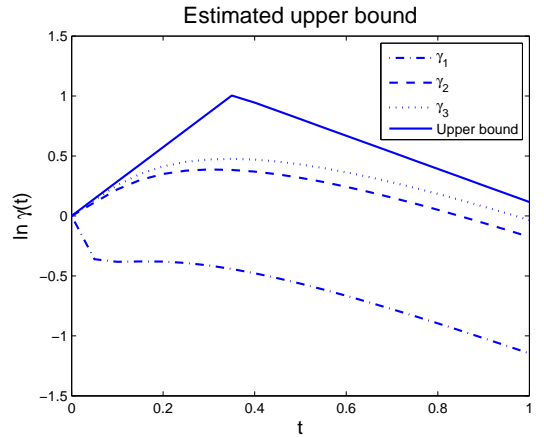

(a) Upper bound

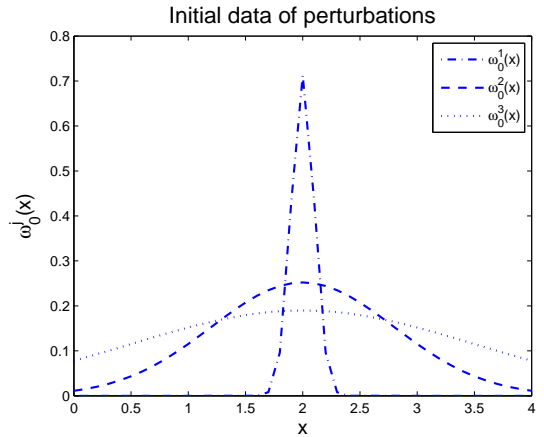

(b) Initial data of perturbations

Figure 2: 2(a): Illustration of Theorem 2.2 with the estimated upper bound and the evolution of perturbations following different initial data in logarithmic scale. 2(b): Initial data of perturbations. Initial data of perturbations $\hat{\boldsymbol{\omega}}_{0}^{1}, \hat{\boldsymbol{\omega}}_{0}^{2}, \hat{\boldsymbol{\omega}}_{0}^{3}$ all satisfy $(3.1)$ with $x_{0}=2$ but with $\delta=0.1,0.8,1.5$ respectively. Parameters are: $J_{11}=-5, J_{12}=-0.5, J_{21}=10, J_{22}=0, d_{1}=$ $1, d_{2}=0.5$.

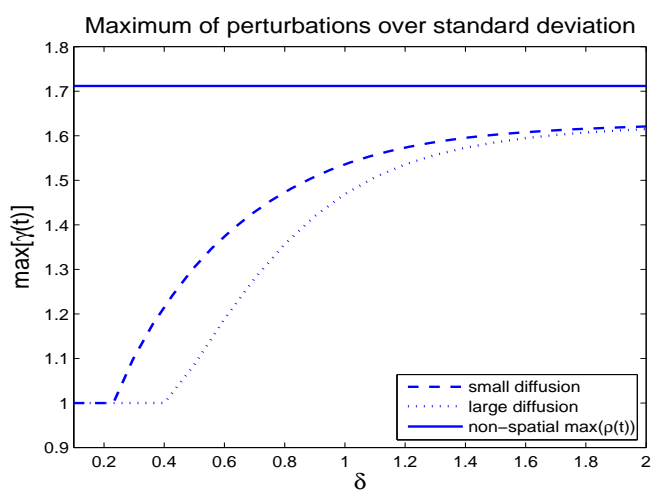

Figure 3: Maximum of $\gamma_{j}$ according to different values of $\delta$ with either small or large diffusion coefficients, and the maximum of the amplification envelope $\rho(t)$ in the corresponding non-spatial model. Parameters are the same as in Figure 2 for plotting the dashed line. Diffusion coefficients are $d_{1}=20, d_{2}=10$ for plotting the dotted line.

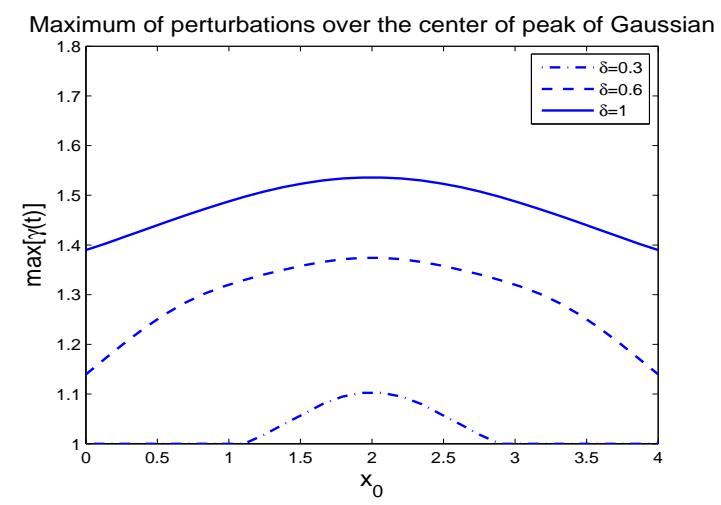

Figure 4: Maximum of $\gamma$ as a function of the center of Gaussian for predator's initial data, according to different values of $\delta$. Parameters are the same as in Figure 2 except $\delta$ in initial data. 


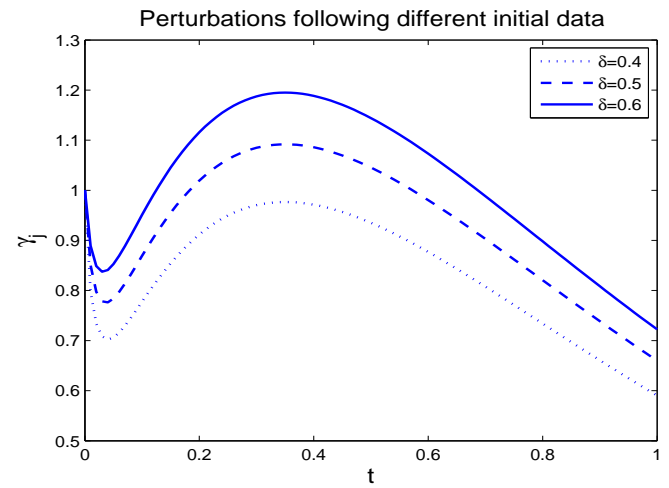

Figure 5: Perturbations following different initial data. Parameters are the same as in Figure 2 except that $d_{1}=20, d_{2}=10$. Initial data satisfy Gaussian with $\delta=0.4,0.5,0.6$ respectively. 